\title{
Pedicle Screw Insertion Surgical Simulator
}

\author{
by \\ Zhechen Du \\ A thesis \\ presented to the University of Waterloo \\ in fulfillment of the \\ thesis requirement for the degree of \\ Master of Applied Science \\ in \\ Electrical and Computer Engineering
}

Waterloo, Ontario, Canada, 2018

(c) Zhechen Du 2018 
I hereby declare that I am the sole author of this thesis. This is a true copy of the thesis, including any required final revisions, as accepted by my examiners.

I understand that my thesis may be made electronically available to the public. 


\begin{abstract}
Scoliosis is a sideway spinal deformity. If the curvature is measured to be more than 50 degrees, the patient can feel significant discomfort. In such cases, surgery is required to straighten the spine. Pedicle screw insertion is a common procedure for scoliosis surgery. The technique requires the placement of screws from the pedicle into the spine. A rod is used to connect all the pedicle screws. The spine is straightened during the connection process. One of the most common techniques used for pedicle screw insertion is called the free hand technique. During free hand surgery, the surgeon creates a screw channel by manually probe into the spine. The lack of visual aid requires the surgeon to rely strongly on haptics feedback. Due to the spine sensitivity and the limited operating range, small changes in force or direction can cause the probe to breach out of the spine. If the breach reaches the spine medial, the spinal cord could be damaged. Even experienced surgeons can not prevent breach. Studies have found that surgeons with 5 or more years of surgical experience have a breach rate of $10.8 \%$.

In this thesis, pedicle screw insertion simulator is developed and examined in detail. The simulator combines visual and haptics sensation to recreate the channel creation process of the surgery. A 2DOF mechanical device is used for the haptics sensation. The device includes a linear actuator and a rotary motor. The simulator was tuned to four different surgical scenarios by 2 expert surgeons. The scenarios are soft probing, hard probing, lateral breach, and in-out-in breach. 10 additional surgeons were asked to participant in a clinical study. Measurements were collected for analysis. The focus of the study is to find if the surgeon can recognize the simulated breach scenarios. Four research questions were examined, and they are: 1 . Can experience help the surgeon improve correct breach recognition rate? 2. Can experience help the surgeon improve overall correct recognition rate? 3. Is there any performance difference between surgeons with different experience
\end{abstract}


level? 4. Can the simulation trials become a learning tool for the simulation tasks? Each question has its own null hypothesis and statistical analysis is used to determine if the null hypothesis is rejected. The main conclusion is that there is no statistically significant relationship between the wrong breach or total wrong recognition rate and surgical experience. Furthermore, there is statistically significant in hard probing scenario between surgical experience and vertical force variance. Lastly, ANOVA analysis showed that the breach force and velocity in three trials are close to statistically significant, more data may prove that the simulator can be a training tool for the tasks. 


\section{Acknowledgements}

I would like to sincerely thank my supervisor, Dr. David Wang, for providing me this opportunity to complete my Master of Applied Science degree. I would also like to thank him for reaching out to Christie Digital in order to provide the funding for the project. Lastly but most importantly, I appropriate his endless knowledge and kind mentorship that helped me completing this thesis.

I also like to thank Dr. Karl Zabjek at University of Toronto. He help coordinated the project between all the interest groups. I also like to thank him for providing necessary space to conduct the study. Lastly, I like to thank him for his valuable input and suggestions during the clinical study phase of the project.

I like to thank Dr. Reinard Zeller and Dr. Ryan O'Shea from Toronto's SickKids Hospital for guiding the simulation tuning process of the project. I would also like to thank Dr. Ryan Katchky for helping recruiting participants to the study.

I like Christie Digital for providing the funding for this project and also their input in the $3 \mathrm{D}$ printed torso design and production.

I thank everyone from my research group on helping me with the project. Special thanks goes to Adam Gomes, Yousef Sawires, Maddie Wang, and Keegan Fernandes for help developing various component of the simulator.

Lastly, I would like to thank Regina Leung and SeyedehMaryam Moafimadani for their previous research and development on the project. Their previous knowledge from the project are essential to the success of the project. 


\section{Table of Contents}

List of Tables $\quad$ x

List of Figures $\quad$ xi

1 Introduction $\quad 1$

1.1 Spine and Scoliosis . . . . . . . . . . . . . . . . 1

1.2 Pedicle Screw Insertion . . . . . . . . . . . . . . . . . 5

1.3 Alternative Surgical Techniques Development . . . . . . . . . . . . . 10

1.4 Surgical Risks . . . . . . . . . . . . . . . . . . . . . . 12

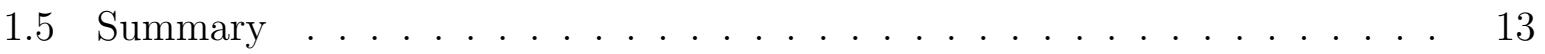

2 Background $\quad 14$

2.1 Existing Training Method . . . . . . . . . . . . . . . . 14

2.2 Virtual Reality ........................ 15

2.3 Virtual Reality Surgical Simulator . . . . . . . . . . . . . . 16

2.4 Existing Research on Virtual Reality Scolisis Surgery . . . . . . . . . . . . 19 


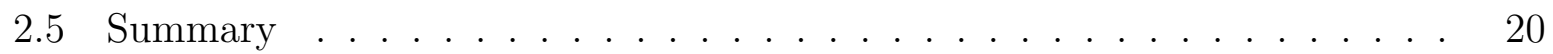

3 Previous Research with the UW Haptic Simulator $\quad 21$

3.1 Rotary Stage . . . . . . . . . . . . . . . . . . . . . . . 23

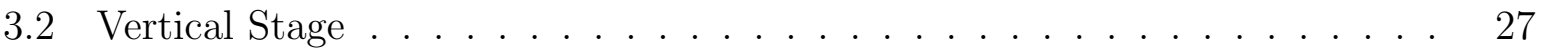

3.3 3D Printed Torso $\ldots \ldots \ldots \ldots \ldots$

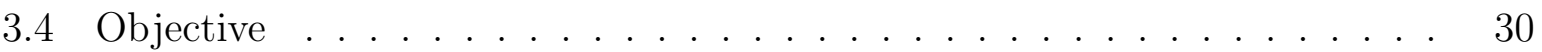

3.5 Summary . . . . . . . . . . . . . . . . . . . . . . 31

4 Linear Actuator Control $\quad 32$

4.1 Actuator . . . . . . . . . . . . . . . . . . . 32

4.2 Actuator Replacement . . . . . . . . . . . . . . . . . . . . . 33

4.3 Actuator Driven Mechanism . . . . . . . . . . . . . . . . . . . . . . . 34

4.4 Normal Probing Control model . . . . . . . . . . . . . . . . . . . . 35

4.5 Breach Simulation . . . . . . . . . . . . . . . . . . . . . . 39

4.6 Linear Control System . . . . . . . . . . . . . . . . . . . . . . . . 45

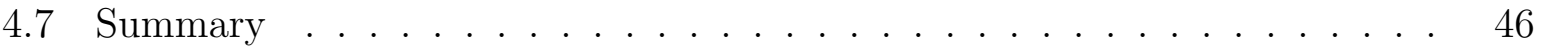

$\begin{array}{llr}5 & \text { Clinical Testing } & 47\end{array}$

5.1 Simulator Tuning . . . . . . . . . . . . . . . . . . . . . 47

$5.1 .1 \quad$ First Session . . . . . . . . . . . . . . . . . 48

5.1 .2 Second Session $\ldots \ldots \ldots \ldots$ 
5.1 .3 Third Session . . . . . . . . . . . . . . . 50

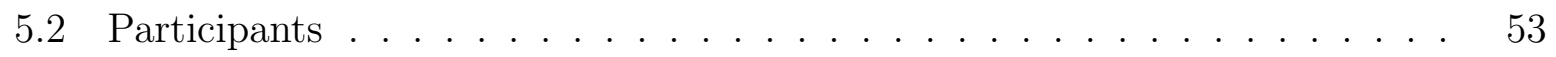

5.3 Experimental Procedure . . . . . . . . . . . . . . . . . 53

5.3.1 Physical Set-up ....................... 53

5.3.2 Training Session . . . . . . . . . . . . . . . . 54

5.3.3 Experimental Protocol . . . . . . . . . . . . . . . 54

5.3.4 Survey Questionnaire . . . . . . . . . . . . . 55

5.4 Research Question ... . . . . . . . . . . . . . . 56

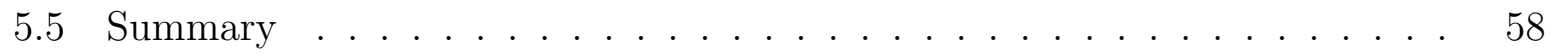

$\begin{array}{llr}6 & \text { Discussion } & 59\end{array}$

6.1 Research Question No. 1 . . . . . . . . . . . . . . . 59

6.2 Research Question No. 2 . . . . . . . . . . . . . . 63

6.3 Research Question No. 3 . . . . . . . . . . . . . . . . 64

6.3.1 Robust Regression Analysis . . . . . . . . . . . . . . 65

6.3.2 Breach Force Variance . . . . . . . . . . . . . . . . . . 71

6.4 Research Question No. $4 \ldots . . \ldots . \ldots 73$

6.5 Questionnaire ....................... 75

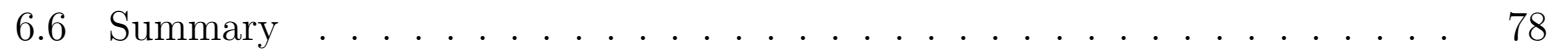


7 Conclusion and Future Works

7.1 Conclusion . . . . . . . . . . . . . . . . . . . . . . . . . 79

7.2 Future work . . . . . . . . . . . . . . . . . 81

$7.2 .1 \quad$ Virtual Project System . . . . . . . . . . . . . . . . . . . . 82

$\begin{array}{lr}\text { References } & 84\end{array}$

$\begin{array}{lr}\text { APPENDICES } & 93\end{array}$

$\begin{array}{lr}\text { A PDF Plots From Matlab } & 94\end{array}$ 


\section{List of Tables}

4.1 Normal probing tuning parameters . . . . . . . . . . . . . . . . 39

4.2 Breach tuning parameters .................... . . . 43

4.3 Linear stage tuning parameters . . . . . . . . . . . . . . 45

5.1 Vertical stage and normal linear stage tuning parameters . . . . . . . . 49

5.2 Breach tuning parameters ........................ 50

6.1 Incorrect identification results from lateral breach and in-out-in breach. . . 60

6.2 Regression analysis results for surgeon's experience vs number of wrong breach identification. . . . . . . . . . . . . . . . . 62

6.3 Total number of incorrect identification results . . . . . . . . . . . . 63

6.4 Regression analysis results for surgeons experience vs total number of wrong identification. ......................... 64

6.5 Robust regression analysis p-value. . . . . . . . . . . . . . . . 70

6.6 One way ANOVA p-value . . . . . . . . . . . . . . . . 75

6.7 Questionnaire result elevation ................ . 77 


\section{List of Figures}

1.1 Side and back view of Vertebrae column[1] . . . . . . . . . . . . 2

1.2 Vertebrae structure[2] . . . . . . . . . . . . . . . . 3

1.3 Normal spine compared with Scolisis[3] . . . . . . . . . . . . . . 4

1.4 Pedicle screw inside the pedicle $[4] \ldots \ldots \ldots$. . . . . . . . . . 5

1.5 Pedicle screw insertion before and after surgery $[5] \ldots . . . . . . .66$

1.6 Pedicle screw insertion procedure. (A) Thoracic vertebra with entry point marked in circle. (B) Posterior cortex removal using a burr. (C) and (D) Probe penetrating though the pedicle showing angulation and inclination. (E) Palpating device inside the spine. (F) Screw insertion inside the channel.

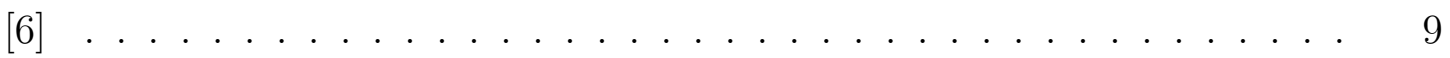

1.7 Pedicle screw insertion surgery using CT [7] . . . . . . . . . . . . 11

2.1 Procedicus MIST, a simulator developed by Mentice for Laparoscopy Surgery

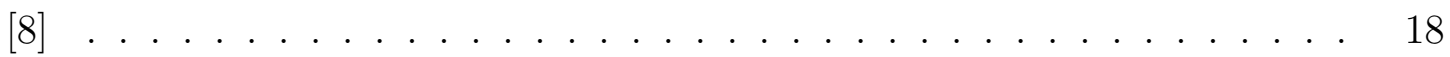

3.1 Surgical simulator after phase two $[9] \ldots \ldots$. . . . . . . . . . . . . 22

3.2 Rotary stage of the surgical simulator[10] . . . . . . . . . . . . 23 


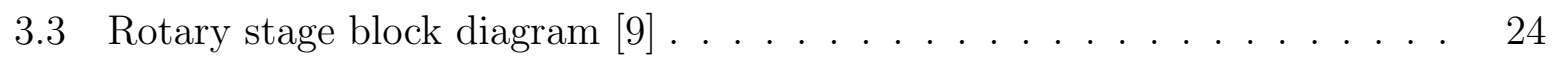

3.4 Rotary proportional controller output illustration $[9] \ldots \ldots \ldots$

3.5 Linear stage of the surgical simulator $[10] \ldots \ldots \ldots \ldots$

3.6 Frequency and duty cycle of the pulses $[9] \ldots \ldots \ldots$

3.7 3D printed torso on the top of the simulator $\ldots \ldots \ldots$

4.1 Electric actuator with driver[11] . . . . . . . . . . . . . . . 34

4.2 Block diagram of the linear actuator control scheme. . . . . . . . . . . 36

4.3 Spring damper model of the pedicle bone. A) Probe drilling into the pedicle layer. B) Spring and damper inside each layer . . . . . . . . . . . . . 39

4.4 Illustration of breach reaching cortex bone of the pedicle . . . . . . . . 40

4.5 Resistance magnitude change with respect to the probe travel length in constant applied force . . . . . . . . . . . . . . . 41

4.6 Resistance magnitude change during breach with respect to the probe travel

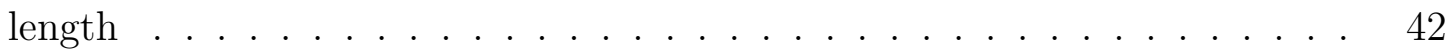

4.7 Resistance magnitude change in probe adjustment scenario with respect to the probe travel length . . . . . . . . . . . . . . . . . . . . 42

4.8 Block diagram of the vertical component . . . . . . . . . . . . . 45

5.1 Four simulation scenarios developed by the surgeons . . . . . . . . . . . 52

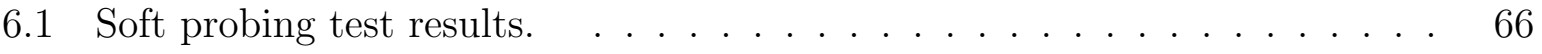

6.2 Hard probing test results. (Participant 6 did not conduct this test) $\ldots .67$ 
6.3 Lateral Breach probing test results. . . . . . . . . . . . . . . . . . 68

6.4 Lateral breach force variance standard deviation. . . . . . . . . . . . . 72

6.5 Lateral breach test results. . . . . . . . . . . . . . . . . . . . 74

6.6 Questionnaire result. ...................... 76

$7.13 \mathrm{D}$ image project on the torso $[12] \ldots \ldots \ldots . \ldots . \ldots . \ldots 2$ 


\section{Chapter 1}

\section{Introduction}

Pedicle screw insertion is a surgical procedure that is used to treat scoliosis. In this chapter, scoliosis and surgical procedure will be presented. The surgical complexity and risk will also be discussed.

\subsection{Spine and Scoliosis}

The human spine, or vertebral column is made up of 33 different bones. The vertebral column can also be separated into upper and lower vertebrae. The 24 upper vertebral are flexible while the lower vertebrae are fused together. The upper vertebral column can further divide into cervical, thoracic, and lumbar vertebrae. They are show in Figure 1.1. The vertebrae are connected though the interverbal disc. Each vertebra is made up of the body, arch and processes. They can be seen in Figure 1.2. Each vertebra arch is a ring shaped structure that can be split into two half arch, each half consists of a pedicle and a lamina. An opening is formed inside the vertebra arch called the vertebral foramen. The 
spinal cord is contained inside the vertebral foramen. The spinal cord is responsible for the connection between the brain and rest of the body. Although there is no clear boundary, the vertebrae body can be separated into cortex bone and cancellous bone based on bone density. The cortex bone is the hard shell on the outside and the cancellous bone is soft bone inside the cortex bone.

\section{Normal Spine}
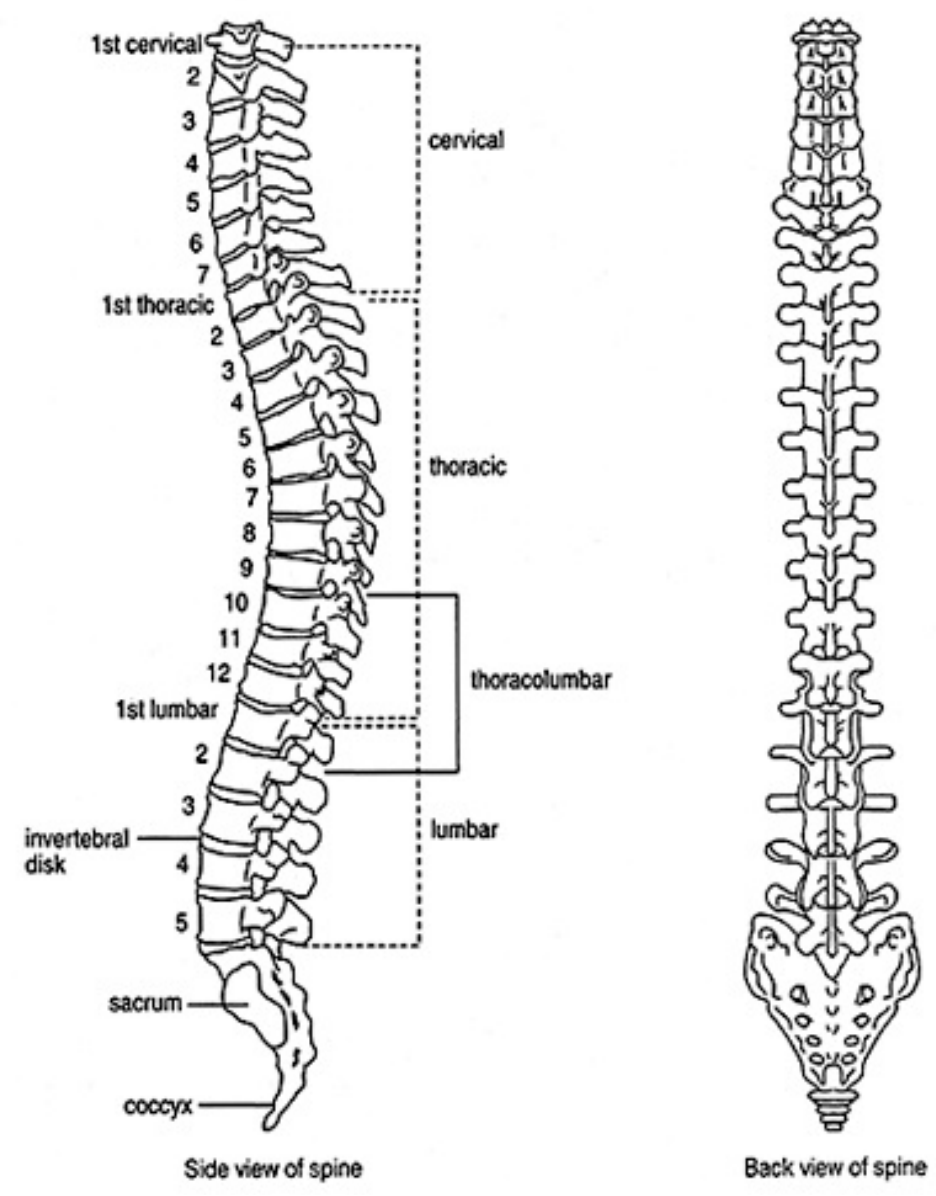

Figure 1.1: Side and back view of Vertebrae column[1] 


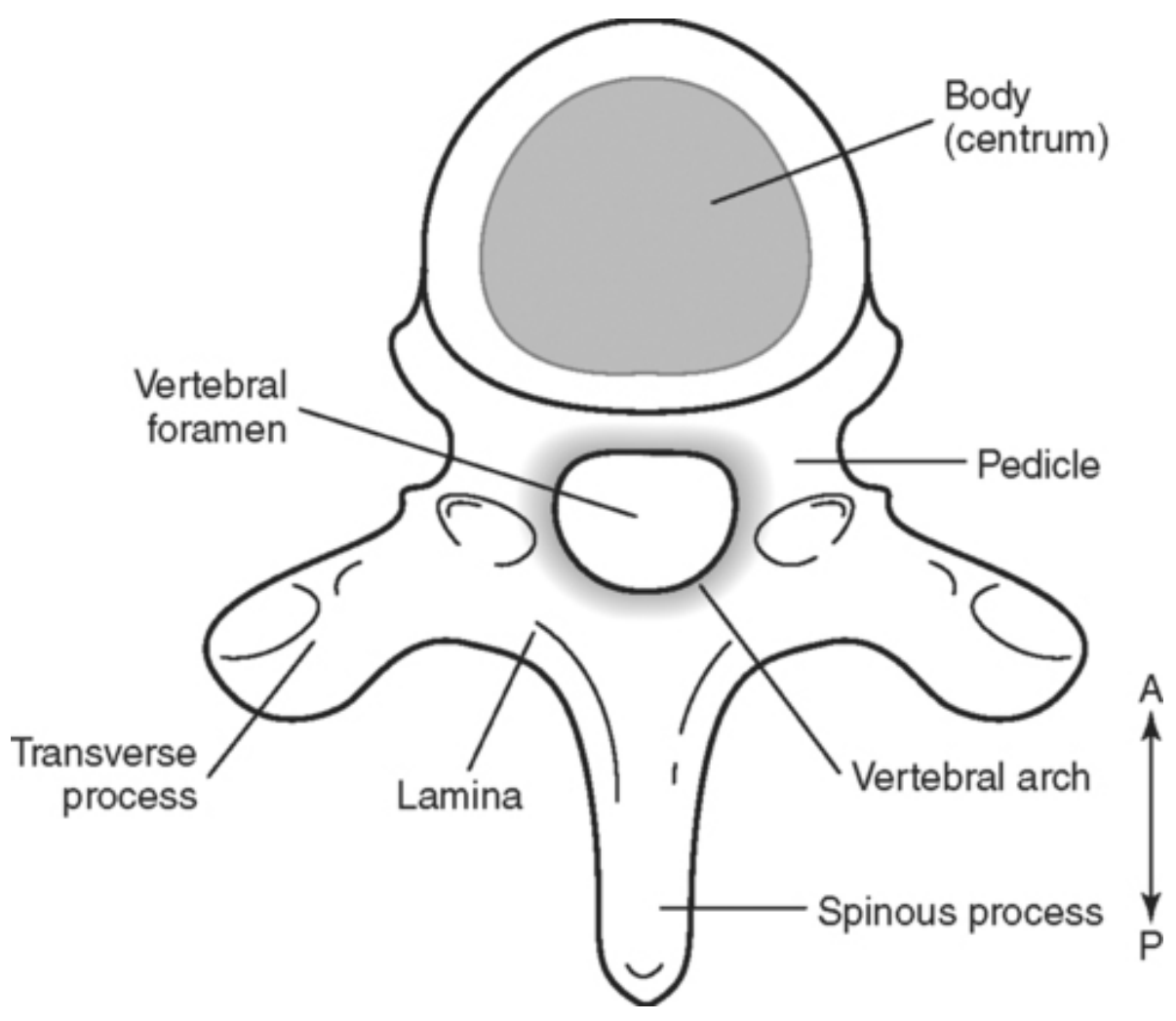

Figure 1.2: Vertebrae structure[2]

The human spine is one of the most stressed part of the human body. In fact, more than 54 percent of adult will experience back pain during some part of their life $[13,14]$. Scoliosis is a sideway spinal deformity. The deformity causes the spine to form into a "S" or "C" shape. Scoliosis is determined if the curvature is bigger than 10 degrees [15]. Scoliosis is a common condition that is usually formed in children before age of 18 , and the process usually occurs during natural human growth [16]. Although some scoliosis can be related to genetic inheritance, about 80 percent of scoliosis has no identifiable cause. The unidentified scoliosis is grouped together as idiopathic scoliosis [15]. Idiopathic scoliosis itself generally does not cause any pain. However, the lateral curvature can cause balance 
issues which can lead to problems such as back pain. Most scoliosis are mild, which do not need treatment. However, the doctor has to constantly observe the patient to see if the scoliosis is becoming more severe. In more problematic cases, the doctor will recommend the patient to wear a brace. This may prevent further spine deformation. If the curvature between any two point of the spine is measured to be more than 50 degrees, the patients can feel significant discomfort. In such cases, surgery is required to straighten the spine $[17]$.

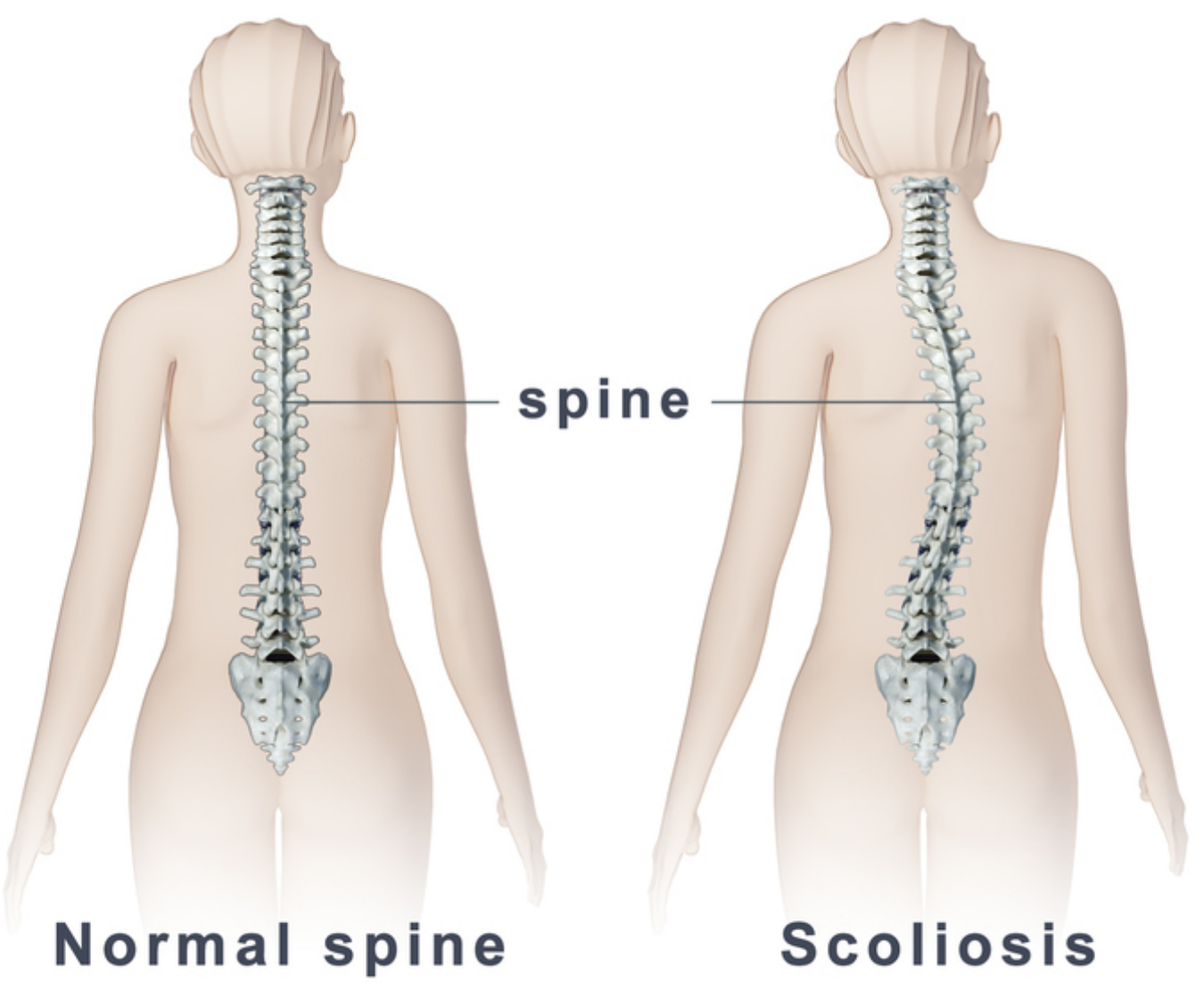

Figure 1.3: Normal spine compared with Scolisis[3] 


\subsection{Pedicle Screw Insertion}

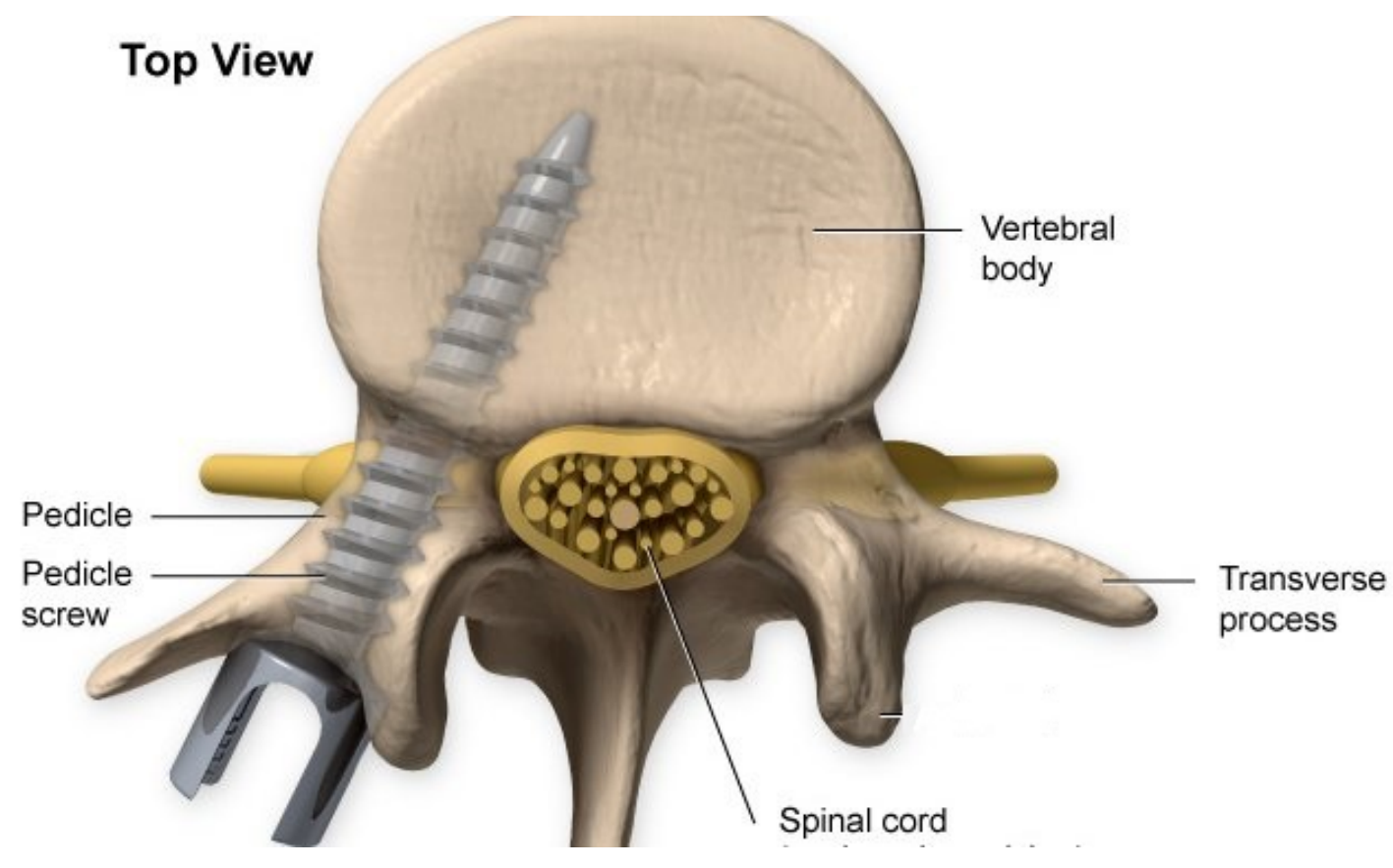

Figure 1.4: Pedicle screw inside the pedicle [4]

Pedicle screw insertion is a common procedure for scoliosis surgery [18]. The surgery is usually performed on the patient after spine growth has stopped. The technique requires the placement of pedicle screws from the pedicle into the vertebral body (See Figure 1.4). The inserted screw is able to connect the anterior, middle, and posterior column of the spine. The three-column fixation technique provides stability and improved curvature of the spine [19]. After all screws are placed into the spine, a rod is used to connect the screws together(See Figure 1.5). Because the pedicle is one of the strongest part of the spine, the rod straightening process rarely causes any bone fracture [20]. Like all spine related surgery, this technique has many risks such as spine fluid leakage, nerve damage, and spine fracture. 


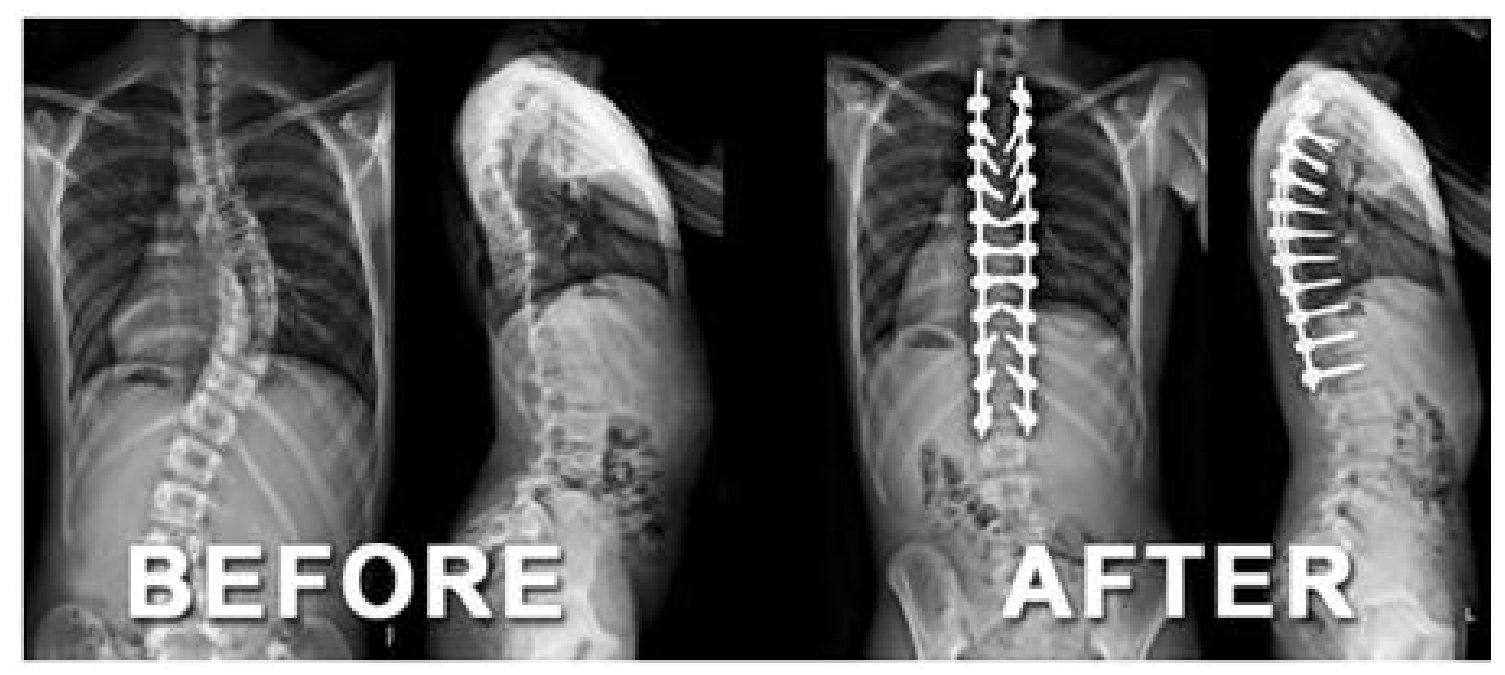

Figure 1.5: Pedicle screw insertion before and after surgery [5]

One of the most common technique used for pedicle screw insertion is called the free hand technique $[18,21]$. During the free hand surgery, the surgeon has to probe manually into the spine without any visual feedback. Because of the lack of visual feedback, this technique requires surgeon to have a clear knowledge of spine structure and landmarks. It also requires tactile haptics feedback, particularly because the bone structure is often deformed due to scoliosis[19]. In order to place the screw into the vertebra, a channel has to be created in the spine first. The following describes the procedure for pedicle screw insertion (see Figure 1.6).

\section{Surgery Preparation}

A clear exposed section of the spine is required for the surgery. Soft tissues and cartilage are removed to limit destruction. The spine should be carefully examined with the help of a CT image. The probe entry point is identified and landmarked after the examination [22]. 


\section{Posterior Cortex Removal}

A burr is used to penetrate through the posterior cortex of the spine. This can help surgeon visualize the starting point of the channel. Because cortex bone is denser than cancellous bone, the probing haptics feedback is different from the cancellous bone probing feedback. Therefore, a two stage cortex drilling is needed to provide a consistent haptics feedback during pedicle probing. Due to the tool change, this part is not part of this thesis.

\section{Channel Creation}

Due to the complexity of channel creation procedure, a simulator is developed as a training tool for the surgery. This is the main focus of this thesis. A curve or straight pedicle probe is then used to channel though the cancellous bone. The probing process should be a smooth and consistent. The surgeon requires haptics feedback to identify the position of the probe. Sudden change in resistance could indicate breaking out of the pedicle bone and into the soft tissue. This event is called a breach [22]. If the breach is in the direction of the vertebral foramen, it is called medial breach. Due to the limited operating space, this is by far the most challenging part of the surgery. After removing the pedicle probe, the surgeon has to make sure only blood is flooding out of the spine. Any other fluid would indicate a breach.

\section{Palpation}

A pedicle sounding probe or palpating device is placed inside the channel to make sure no breach has occurred. If a breach is identified, the channel has to be reexamined In most cases, the breach does not cause any damage and can be salvaged by using different techniques. In more serious situations, bone wax is placed inside the pedicle to stop bleeding, and a new trajectory has to be channelled inside the 
pedicle [22].

\section{Screw Placement}

The channel diameter depth is measured to determine the screw size. After all screws are inserted into the spine, a rod is used to connect the screws to fix the spine curvature. The screws are secured after the rod position is fixed. In most of the cases, the screw and the rod will permanently attached to the spine. 

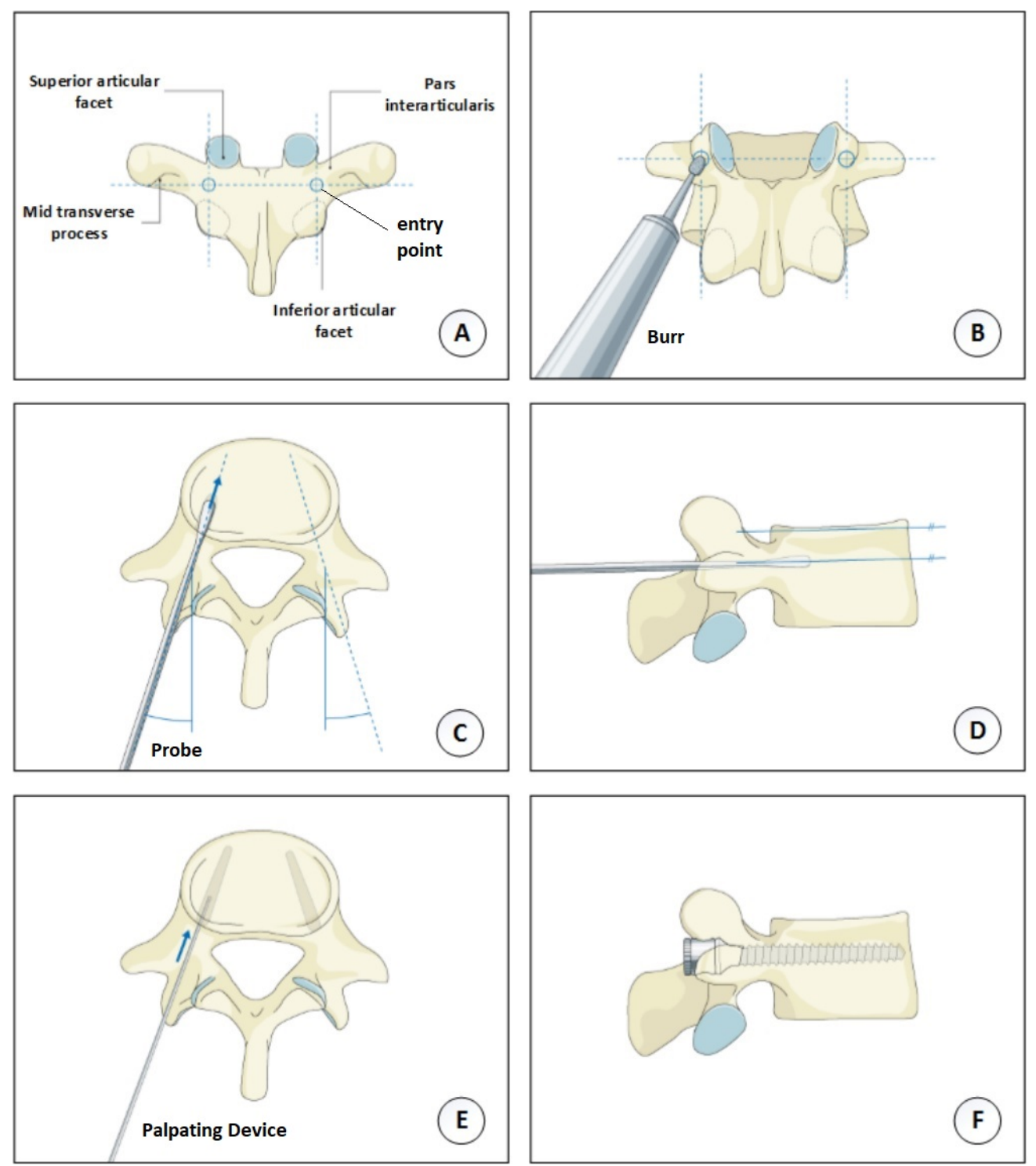

Figure 1.6: Pedicle screw insertion procedure. (A) Thoracic vertebra with entry point marked in circle. (B) Posterior cortex removal using a burr. (C) and (D) Probe penetrating though the pedicle showing angulation and inclination. (E) Palpating device inside the spine. (F) Screw insertion inside the channel. [6] 


\subsection{Alternative Surgical Techniques Development}

More recently, computer aided surgery has become more popular. 3D fluoroscope and CT have been adopted to reduced surgery complexity for surgeons. In almost all of the surgical procedures, the surgeon uses CT images before the surgery. This allows the surgeon to plan possible entry points and trajectory lines. Computer aided technology has also been adopted during surgery. The surgeon is able to use virtual images to follow trajectory lines and avoid possible breach. an illustration of such procedure is shown in Figure 1.7. Studies have shown that computer aided methods generally provides higher accuracy and lower risks [23, 24]. However, there are drawbacks such as a higher learning curve and operation cost. Due to the scanning time and adjustment during probing, the operation time is much longer compared to free hand probing. The constant exposure of radiation during surgery is also another concern for the surgeon. The mean radiation exposure for the fluoroscopy procedure is $4.33 \pm 2.66 \mathrm{mRem}$ and the $\mathrm{CT}$ procedure is $0.33 \pm 0.82 \mathrm{mRem}$ [25]. If a surgeon is constantly performing complex procedures, fluoroscope radiation exposure may exceed annual accumulation limits [26]. Because CT imaging is not real time and requires extra operation time, surgeon are sometimes forced to use fluoroscope. Moreover, outside of imaging assessment, there is currently no report on how patients recovery is affected by the new technology [27]. Moreover, case study literature reviews rarely identifies any major neurological and vascular injury [18]. This further complicates the evaluation of the surgery. 


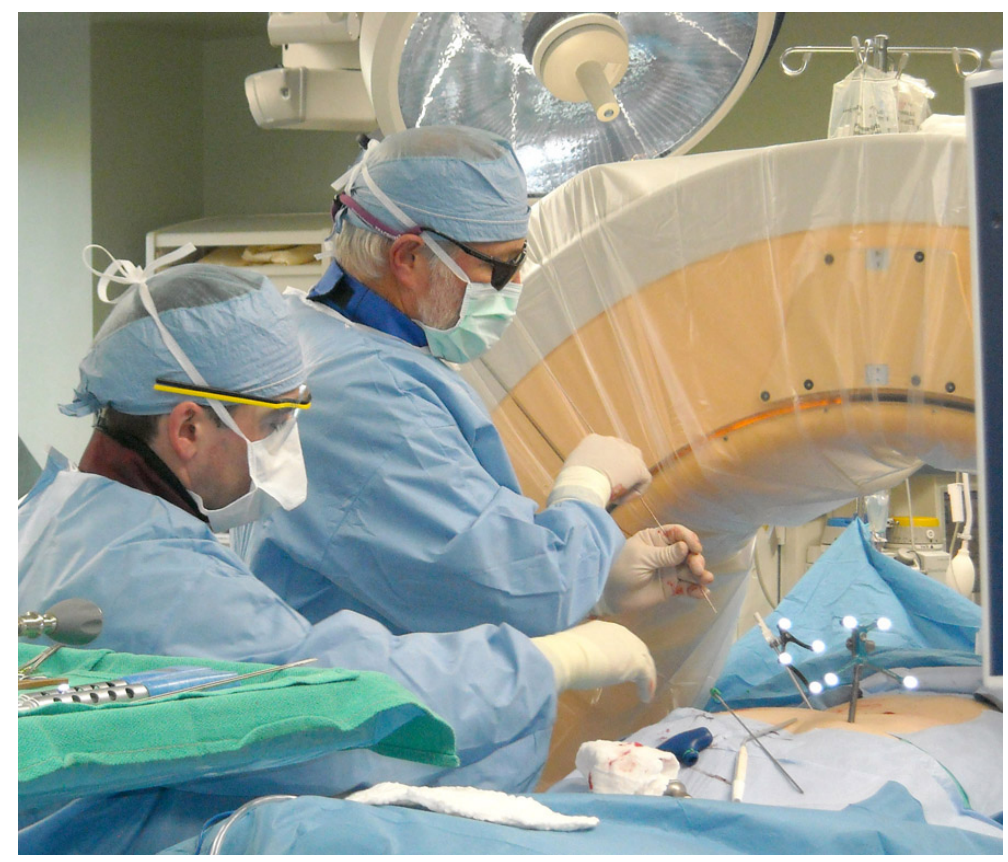

Figure 1.7: Pedicle screw insertion surgery using CT [7]

Robotic guidance placement has also become a recent research topic [28]. From the presurgery CT scan, the robot is able to determine possible drilling points and trajectories. During the surgery, a guided drill is fixed on to the patient. The surgeon is able to use the fixed drill for the insertion process. This can remove any human error during channel creation. Due to its complexity, the robot guided procedure requires far more time to complete. Furthermore, a study has found that conventional free hand techniques are still safer [29]. 


\subsection{Surgical Risks}

Due to the high fragility of the spine, the pedicle screw insertion surgery comes with great risks. Any mistake could cause severe problems for the patient. The surgery is further complicated by the structure of the spine with respect to the probing location and the deformity of the spine. Due to this, each surgery is unique to the deformity. Furthermore, due to the limited operating range of the probe inside the pedicle, small changes in force or direction could cause a breach. If the breach reaches the spine medial, the spinal cord could be damaged, which would be disastrous for the patient. Because there is no visual observation, the surgeon must depend strongly on haptic feedback from the probe. This challenges surgeons mentally and physically to make the correct decisions during the probing.

There have been many studies on the accuracy of pedicle screw insertion. In one review conducted from 21 studies with 4570 pedicle screws in 1666 patients[18], the reported screw misplacement rate is around $11 \%$. Revision surgery was performed on 12 patients for misplaced or loose screws. In another review, the reported accuracy rate from different studies for free hand pedicle screw insertion ranged from $69 \%$ to $94 \%$ [24]. This clearly shows incorrect surgical practice can bring a lot of damage to the patient.

Surgeon experience is an important factor that can affect the accuracy of free hand pedicle screw insertion. In a study conducted by Samdani et al. [30], 856 samples was collected and reviewed. The breach rate for surgeons with less than 2 years experience is $12.7 \%, 2-5$ years experience is $12.9 \%$, and 5 or more years of experience is $10.8 \%$. There is no statistically significance between the three groups. However, for more serious medial breach, the breach rate for surgeons with less than 2 years experience is $7.4 \%, 2-5$ years experience is $8.4 \%$, and 5 or more years of experience is $3.5 \%$. This is correlated by 
another study conducted by Lehman et al. [31], where it has shown that the surgeon's medial breach rate using the free hand technique decreases over an 8 year period.

Although experience of the surgeon can reduce risks, even the most experienced surgeon cannot prevent breach. One particular study analysed the accuracy rate of 2 surgeons with more than 10 years experience. In this study, $6.2 \%$ of the screws were malpositioned and $1.7 \%$ screws were considered to be medial breach [32].

\subsection{Summary}

This chapter introduced spine and scoliosis surgery. Pedicle screw insertion sugery and the free hand technique are explained. Lastly, the surgical risks and other surgical techniques are discussed. 


\section{Chapter 2}

\section{Background}

In this chapter, the existing industry training approaches for scoliosis surgery will be examined. Furthermore, virtual reality and research of using VR for scoliosis surgery will be discussed.

\subsection{Existing Training Method}

The complexity of spine surgery requires the surgeon to be well trained before they perform scoliosis surgery. In the past, there were two main ways to prepare for the surgery. They are cadaver training and apprenticeship in the operating room.

Training on cadavers is one of the most realistic way to practice the surgery. This is because the close resemblance between cadavers and live patients. It is also a very good way to familiarize the surgeon with the surgical procedure. However, the cadaver probing process provides a different haptic feedback sensations compare to a real patient [33]. This is because cadaver is treated with an embalming chemical to prevent decomposition. The 
embalming chemical can change the material property of the bone. This makes the tactile sensation different compare to that of a live patient. Furthermore, the lack of blood and other body fluids can also affect the haptic sensations of the surgery [34]. In most cases, the cadaver does not suffer from spine deformity as spinal deformity changes the spine structure. Thus, regular cadavers can limit the training effectiveness. Lastly, there is limited access to cadavers, which further affects training.

The second approach to training is by apprenticing in the operating room. This follows a traditional training concept know as "see one, do one, teach one" [35]. This model can help the trainee in getting access to the real surgical environment. It is also beneficial for a trainee to receive feedback about their progress during the surgery. One obvious drawback for this approach is the additional risk for the patient. In one study, a spinal surgery fellow's misplacement rate for the first 80 screws was $12.5 \%$. The misplacement rate dropped to $3.3 \%$ for the next 240 screws [36]. It is also noted that high and consistent repetition is required for the apprentice to acquire the surgical skill. Due to the limitation of the trainee's and expert's time, apprenticeship may not be as effective in complex surgeries such as the scoliosis surgery.

\section{$2.2 \quad$ Virtual Reality}

Virtual reality is a computer based technology developed to create an realistic environment by manipulating human sensations. The two main sensation for surgical VR simulator are visual and haptics. Because of todays technology advancements such as the computer and the smartphone, visual sensation is the more studied field. In most scenarios, visual sensation provides reliable feedback information for the operator[37]. However, due to the blind nature of scoliosis surgery, the visual sensation is limited in providing effective 
feedback for scoliosis surgery.

Haptics is the sense of touch that provides lots of information content. Haptics information is received from two different receptors: cutaneous receptors and kinesthetic receptors [38]. The cutaneous receptors are located in or under the skin surface. This receptor allows the human to receive information such as temperature, vibration, and pain. It has a resolution up to $1 \mathrm{kHz}[38]$. The kinesthetic receptor provides feedback of force and torque [39]. It provides awareness of body position and movement. The kinesthetic receptors have a resolution of 2 degrees in the fingers and wrist, and 1 degree of resolution in the shoulder area [39]. Haptics usually has a relative low accuracy as compared to other sensation [34]. The error correction requires a great deal of processing from the brain. Due to its complexity, haptics is still a relatively young field of research. Without visual aids, haptics is especially important in pedicle screw insertion. Surgeons have to be trained to accurately identify the sensation felt through the probe.

\subsection{Virtual Reality Surgical Simulator}

Virtual reality is used extensively in surgical simulation and training. Many surgeons have adopted a learning concept called "see one, simulate many, do one competently, and teach everyone" [35]. In many surgeries, 3D CT or MRI scans are used by the surgeon to help visualizing the surgical procedure. In field where haptics feedback is required, force/torque feedback devices such as the Phantom have been used in addition to visual sensation. Many of such device is described below.

Laparoscopy surgery or minimally invasive surgery is a surgical procedure where operation is completed through small incisions. The location of the operator is generally

far away from the incision. Due to the limited operating space through the incision and 
small force/toruqe feedback, surgeons usually require extensive training to master the procedure. A number of different training simulators have been developed for laparoscopy surgery. One of the first such simulator, Procedicus MIST, was developed by Mentice [34]. As shown in Figure 2.1, the simulator utilizes two computer monitors for visual feedback. In addition, Xitact ITP and IHP devices are used to provide haptics feedback. The IHP is able to provide axial force and pitch, yaw, roll torque [34]. Varies studies have shown that the Procedicus MIST is an effective training tool for laparoscopy surgery [40, 41, 42]. 


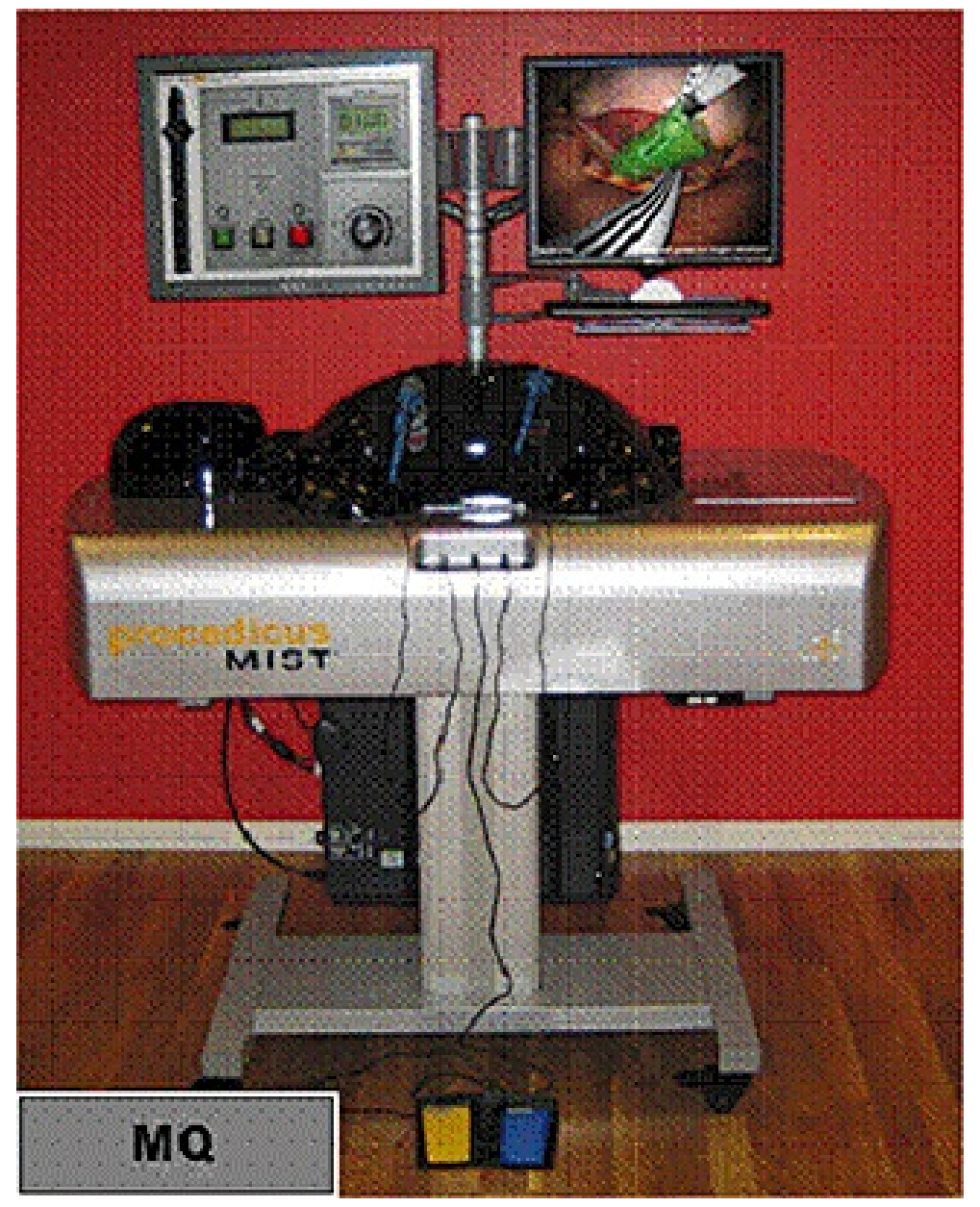

Figure 2.1: Procedicus MIST, a simulator developed by Mentice for Laparoscopy Surgery [8]

Endovascular surgery is a image guided procedure done inside the blood vessel. The surgery requires surgeon to use subtle haptics feedback to direct the guidewire and catheter inside the blood vessel. Training simulators for such surgery includes VIST, ANGIO Mentor, and CathLabVR [34]. Many studies have proved the effectiveness of such simulator $[43,44]$. 
The Geomagic Phantom has also been utilized in providing surgical haptics feedback. For example, there are extensive use of haptics simulation in temporal bone drill training. Wiet et al. [45] developed an platform that utilizes two Phantoms to simulate drill and suction feedback. The platform has since been used as a training tool by many otologists $[46]$.

More recently, Vankipuram et al. [47] developed an orthopedic surgery simulator. In addition to a visual bone model, the surgical simulator used a modified Phantom device to simulate the drilling process. The study has shown that the simulator can provide positive impact in a real surgical environment.

\subsection{Existing Research on Virtual Reality Scolisis Surgery}

In the existing research of pedicle screw insertion simulation, most studies have focused on visual simulation. Many have adapted the use of 3D CT scan as part of the training and surgery. Currently, hundreds of different computer assisted surgery methods have been developed by various universities and institutions [48]. Different 3D models are used to help the trainee to better understand the surgery.

A CT based screw placement simulator has been developed by Klein et al. [49]. In this simulator, a 3D spine model is constructed using patient's CT scan. The trainee can use the program to practice positioning of the screw. A screw placement grading system is developed based on screw position and length. This program helps trainees recognize basic techniques used in pedicle screw placement. Unfortunately, the system has no ability to simulate the channel creation process.

Luciano et al. [50] have developed a simulator that combines haptics with virtual 
reality. Same as Klein's work, a CT scan is used to form the basic model for 3D spine. As for the haptics sensation, the group used an ImmersiveTouch haptic stylus to create force feedback. A lumber Sawbones spine model was used to evaluate the effectiveness of the simulator [51]. Although the haptics feedback force is minimal, the study did show the simulation can be helpful for training a non-expert in complex tasks [52].

In another study, Xing et al. [53] used the Phantom haptics device as the probe to simulate the pedicle insertion process. Spine modelling and collision detection are two important aspect of this paper. However, there was no clinical testing for this approach.

Various other works have been done in this field. For example, Schmidt developed a vertebrae simulation model for power drilling. The model is able to reconstruct CT data after the simulated drilling. In another case, Fang et al. [54] developed a training system to help identify the best probe entry point. An expert surgeon is asked to identify the best

entry point based on his/her judgement. Based on this point, the system will evaluate trainee's picked position.

The main drawback of the existing haptics simulators is the lack of large force simulation. In order to simulate breach, a sudden change in force and velocity is required. All the existing simulators are unable to provide enough force in such an event. This research is motivated due to this problem.

\subsection{Summary}

In this chapter, the existing industry training approaches for scoliosis surgery are discussed. Furthermore, virtual reality and research of using VR for surgery simulation is discussed. Lastly, the existing research on scoliosis surgery simulation is examined. 


\section{Chapter 3}

\section{Previous Research with the UW Haptic Simulator}

This chapter describes the previous work done here at the University of Waterloo in conjunction with SickKids Hospital. An illustration of the simulator is shown in Figure 3.1. The research is focused on developing haptics feedback simulation of the probe navigating through the pedicle. Two researchers have previously worked on the project. Leung started the project by developing the rotary stage of the simulator [10]. Moafimadni conducted the second stage of the research [9], where the vertical stage of the simulator was developed. The rotary stage provides surgeon with a sense of pedicle density and vertical stage can simulate the depth of the pedicle. From surgeon's feedback, it was determined that the 2DOF simulator can provide a realistic haptics simulation for the surgeons. In addition, a 3D printed torso is used to help surgeons better visualize the surgical environment. 


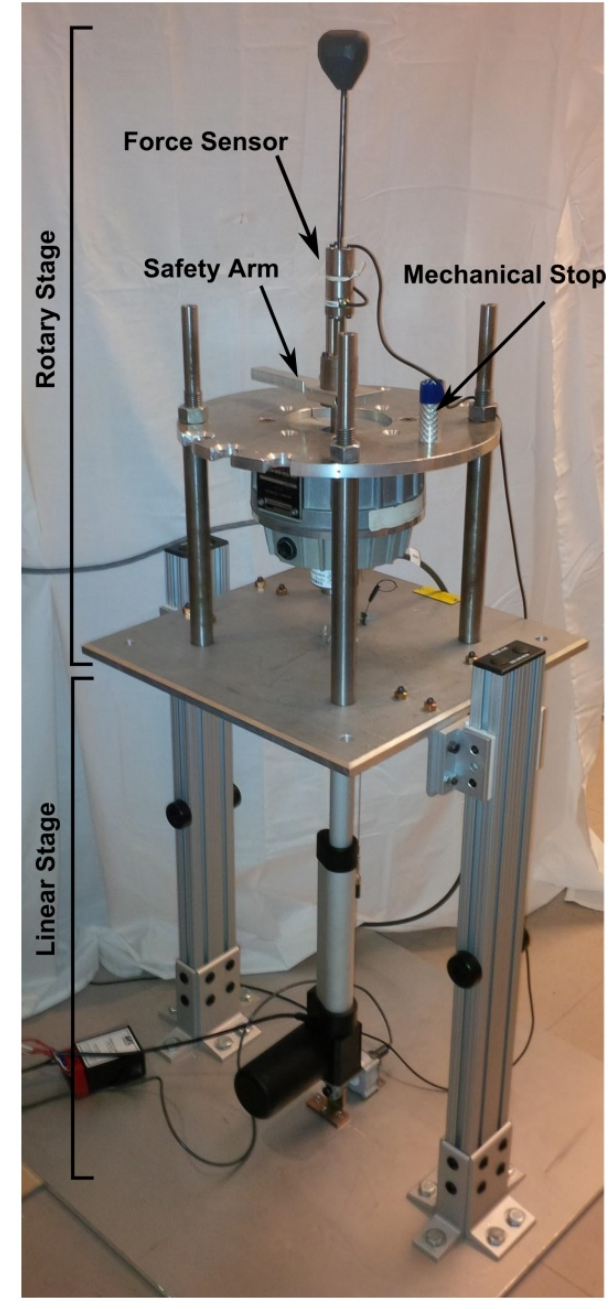

Figure 3.1: Surgical simulator after phase two[9] 


\subsection{Rotary Stage}

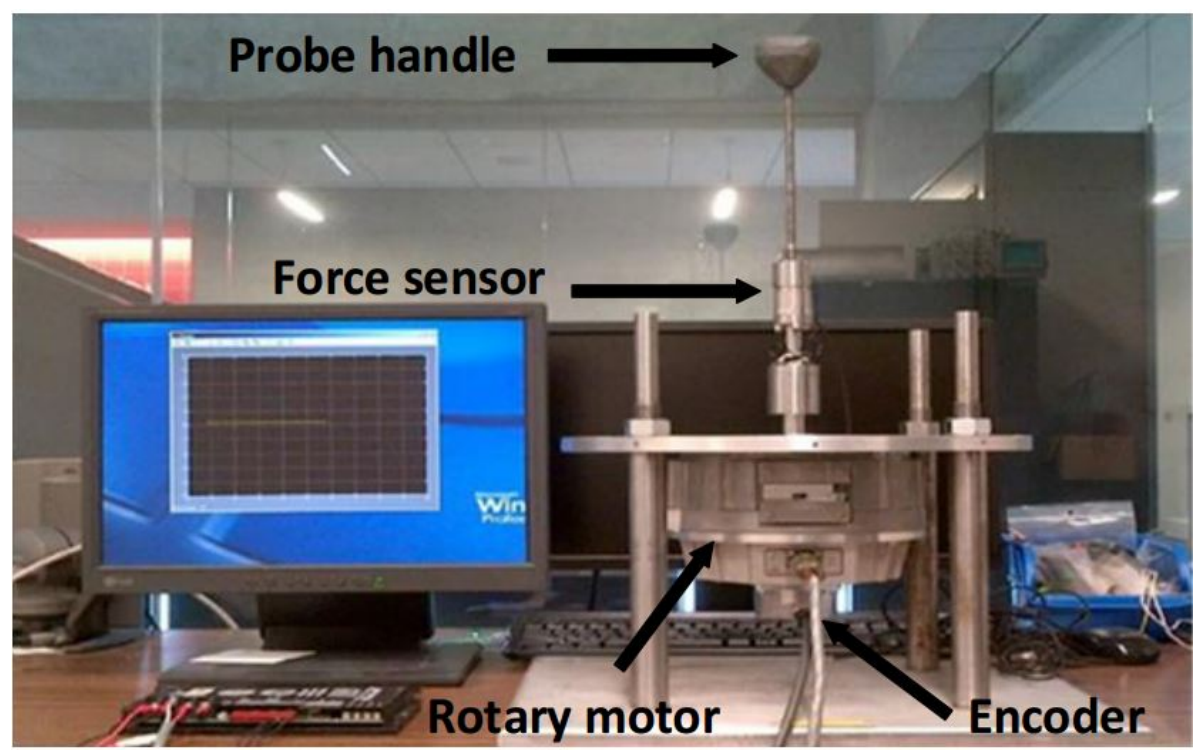

Figure 3.2: Rotary stage of the surgical simulator[10]

The rotary stage of the research on the haptics simulator involves designing and building a 1DOF haptics simulator for probe channelling during pedicle screw insertion surgery[10]. An illustration of the rotary stage is shown in Figure 3.2. The simulation is performed by the surgeon from a probe at the top of the simulator. Ideally, the surgeon should operate the probe matching the real pedicle insertion surgery. A force sensor is attached to the bottom of probe. It is used to measure the surgeon's applied force and torque on the probe. A servo DC motor is positioned at the bottom of the simulator. The motor is used to simulate the force and torque feedback of the probe's rotational movement inside the pedicle. The haptics control model uses four parameters to generate the vibration and viscous friction effect of the pedicle. The parameters are listed as following and will be discussed later: 
- Detent interval $\theta_{i}$

- Detent width $\theta_{w}$

- Detent magnitude $k_{p}$

- Viscous friction coefficient $k_{d}$

Due to its effectiveness, the rotary control model of the simulator is still used in the current design. The tuning procedure of the four parameters can be found in Chapter 5 . A block diagram of the rotary stage is shown in Figure 3.3.

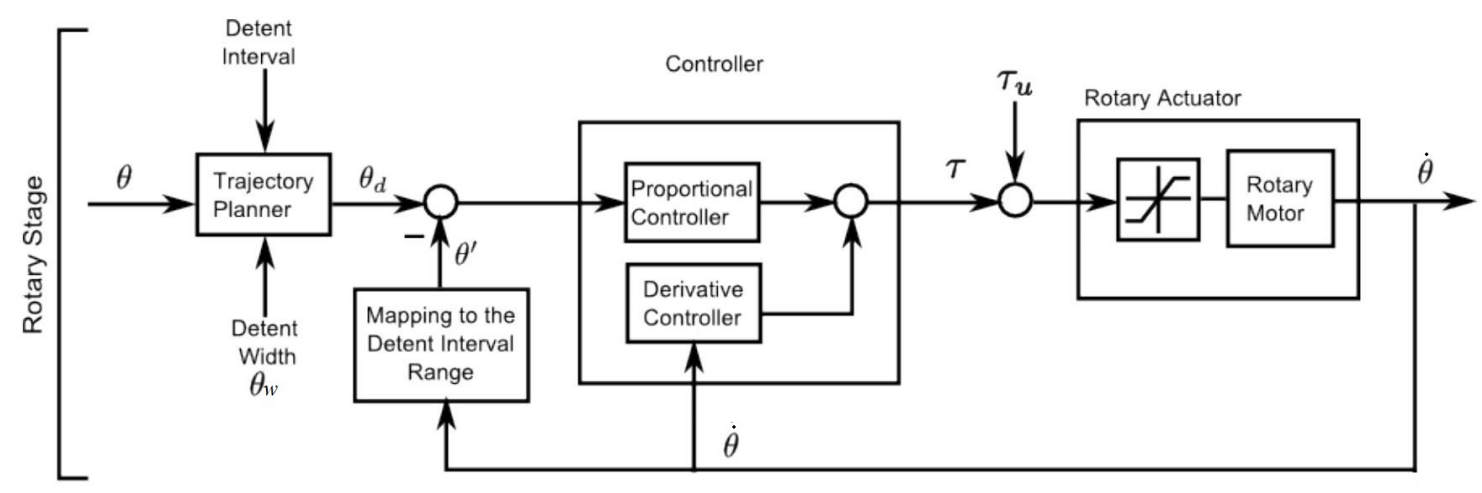

Figure 3.3: Rotary stage block diagram [9]

As shown in the block diagram, the trajectory planner is a piecewise equation that can be adjusted by two user defined inputs: detent interval, $\theta_{i}$ and detent width, $\theta_{w}$. The third input to the trajectory planner is the rotary angular position $\theta$. The trajectory planner 
will output the desired angular position $\theta_{d}$. The piecewise equation is stated as follows:

$$
\theta_{d}= \begin{cases}\theta^{\prime} & \text { if } \theta^{\prime}<\theta_{L} \\ \theta_{L} & \text { if } \theta_{L}<\theta^{\prime}<\frac{\theta_{i}}{2} \\ \theta_{R} & \text { if } \frac{\theta_{i}}{2}<\theta^{\prime}<\theta_{R} \\ \theta^{\prime} & \text { if } \theta^{\prime}>\theta_{R}\end{cases}
$$

where $\theta^{\prime}$ is the remainder of $\theta$ divided by $\theta_{i}$. $\theta_{L}$ and $\theta_{R}$ are calculated using the equation below:

$$
\begin{aligned}
& \theta_{L}=\frac{\theta_{i}-\theta_{w}}{2} \\
& \theta_{R}=\frac{\theta_{i}+\theta_{w}}{2}
\end{aligned}
$$

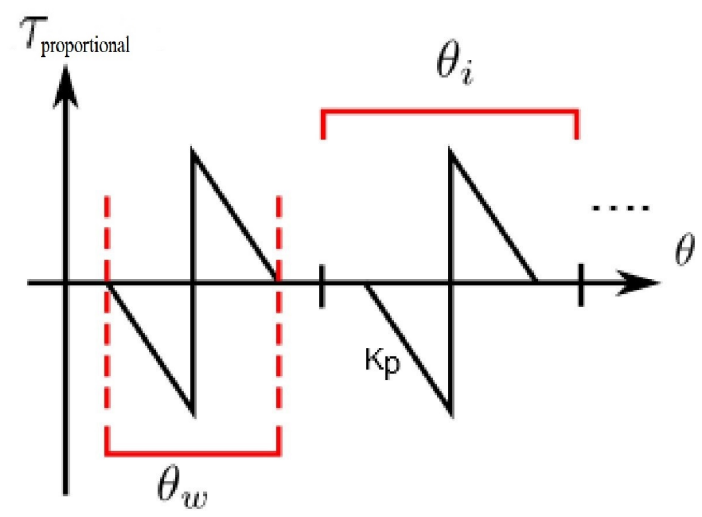

Figure 3.4: Rotary proportional controller output illustration [9]

A PD controller is used to control the output of the controller. In this case, $k_{p}$ is the detent magnitude, and $k_{d}$ is the viscous friction coefficient. The proportional component is shown is Figure 3.4. The equation for this controller is stated as follows:

$$
\tau=k_{p}\left(\theta_{d}-\theta^{\prime}\right)-k_{d} \dot{\theta}
$$


where $\tau$ the output torque is used to control the rotary motor system.

A pilot study was conducted with the help of 5 expert spine surgeons. The surgeons were asked to tune the four parameters to three different feedback levels (High, Medium, Low). From the data collected in the study, there is noticeable difference between surgeons across all three levels. In particular, viscous friction coefficient $k_{d}$ has a large variation. The surgeons were then asked to give a score on the realism of the simulator and potential of using as a training tool. All surgeons at least gave a score of $4 / 5$ for both questions.

Due to the insufficient sample size and lack of clear definition of the three feedback levels, the results can only be used as a reference for the feasibility of the project. The lack of a vertical stage may also affect the haptics sensation felt by the participant. 


\subsection{Vertical Stage}

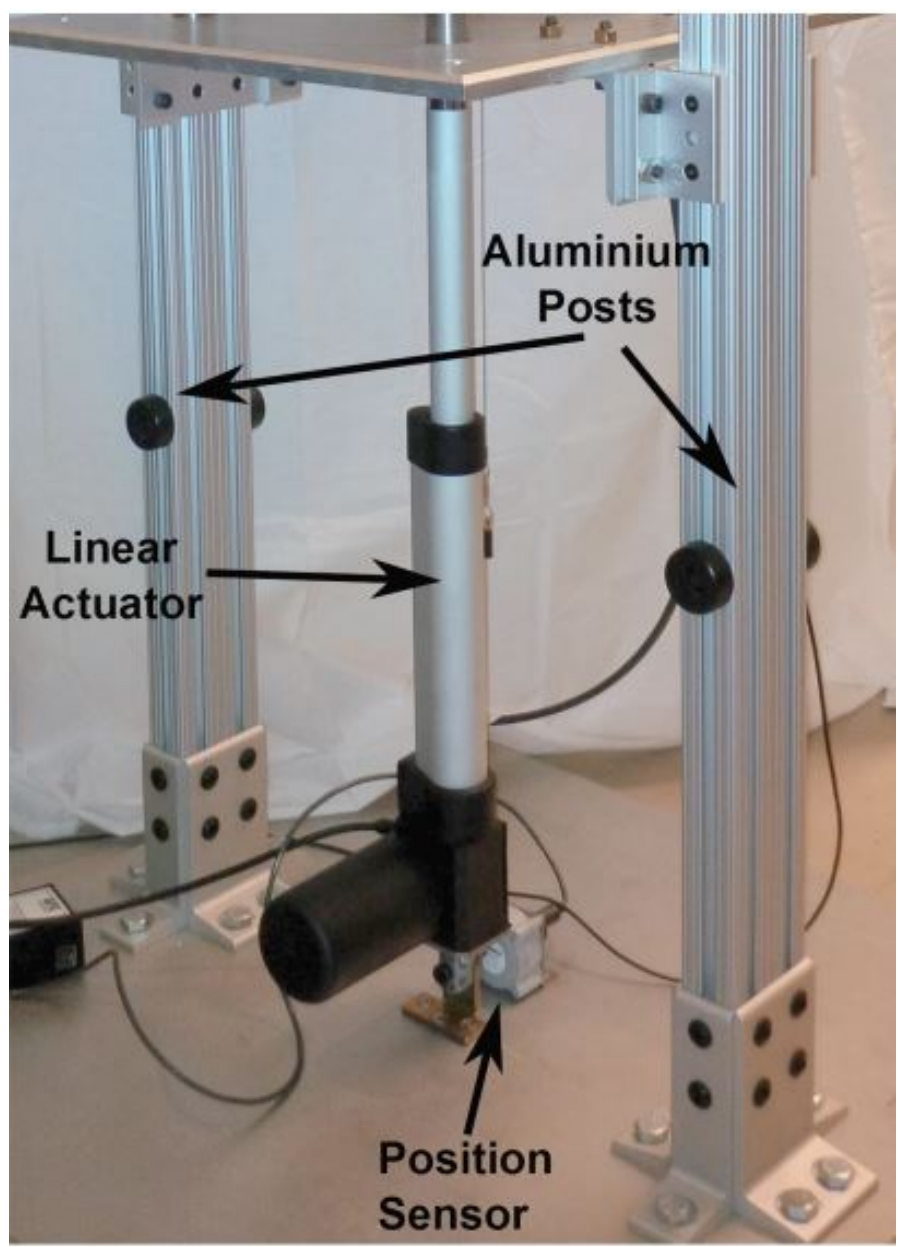

Figure 3.5: Linear stage of the surgical simulator [10]

Based on surgeon's feedback from the tuning study, the lack of vertical movement can affect the surgeon's judgement. The vertical movement is essential in complex scenarios such as lateral breach. To solve this problem, a 2DOF simulator was developed [9]. A linear actuator is added underneath the rotary stage to provide the vertical movement. The 
linear stage is shown in Figure 3.5. The actuator is controlled using open loop technique with three control parameters: frequency, scaling gain, and duty cycle. The input of the controller is user's applied force. The controller is able to transform the force into a series of square wave pulses. The square wave pulses are the applied voltage $(\mathrm{V})$ of the actuator which is used to simulate the vibration inside the pedicle. The frequency is number of the pulse per second and the duty cycle correspond to the pulse width. The velocity of the actuator is determined by the scaling gain. An illustration of the pulses can be found in Figure 3.6.

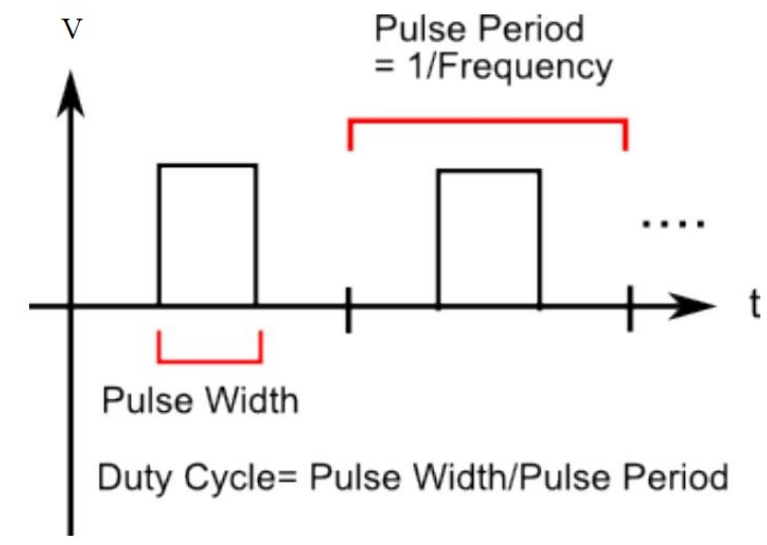

Figure 3.6: Frequency and duty cycle of the pulses[9]

A clinical study was conducted with 11 surgeons. The surgeons consist of 2 seniors, 4 fellows, and 5 residents. The surgeons were asked to tune the rotary and linear stage parameters based on normal healthy spine. The data is used to compare the surgeon's performance with respect to experience level. When comparing to residents and fellows, the study found that senior surgeons had relatively small tuning variation in the following parameters: detent interval, detent magnitude, viscous friction coefficient, duty cycle, and 
frequency of vibration. The null hypothesis of the analysis is stated as following: The mean is equal across surgeons with different expertise levels. ANOVA and Welch test were conducted on the control parameters. Two parameters (detent magnitude and frequency) have significance values that are close to statistically significant. With a bigger sample size, the study could possibly reject the null hypothesis and prove that experience can affect the parameter tuning. The parameters may potentially be used to evaluate a surgeon's performance. Based on the survey questionnaire, all seniors and fellows found the simulator to be a useful training tool for pedicle screw insertion.

One of the major limitations for this simulator is the linear actuator power. It is not feasible to implement closed loop control due to the slow reaction time. The open loop control system can present accuracy issues as there is no way to control the actuator based on the current position. This may affect the feasibility of simulating complex scenarios. Furthermore, breach simulation was also not possible due to the slow actuator speed. Lastly, the lack of a visual aid can also affect the realism of the simulation. This could potentially limit surgeon's judgement during the study. 


\subsection{D Printed Torso}

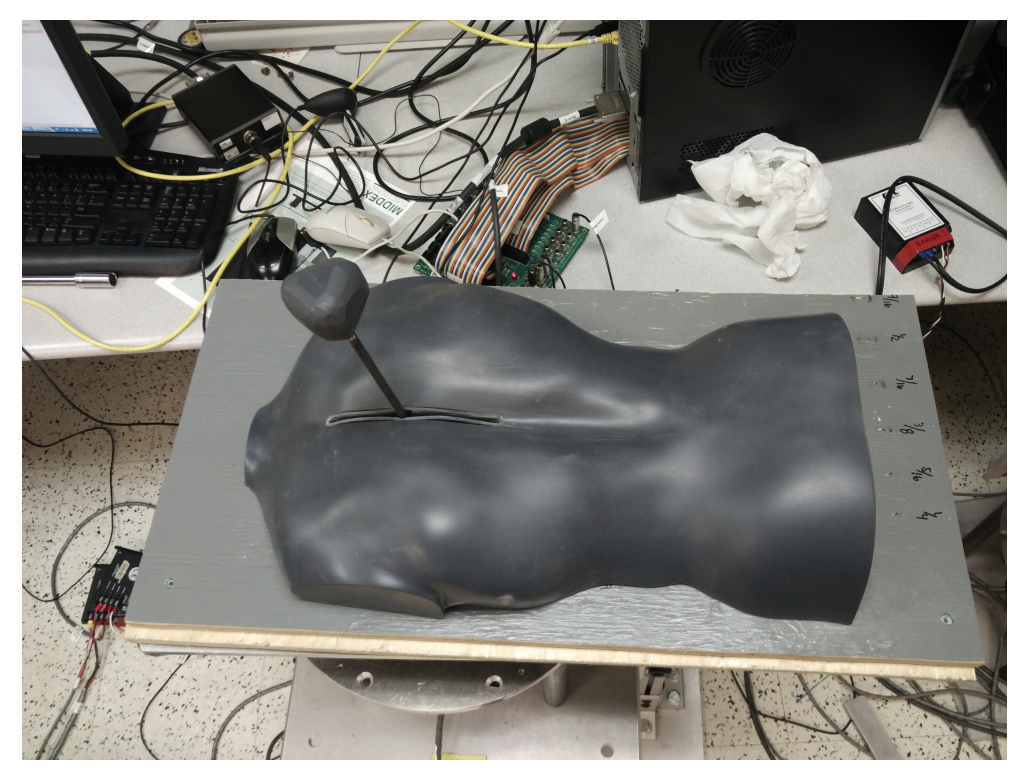

Figure 3.7: 3D printed torso on the top of the simulator

In order to improve the realism of the actuator, a 3D printed torso is used to provide visual aid to the surgeon. The torso is placed at the top of the simulator. The surgeon is able to push on the torso similar to a real surgery.

\subsection{Objective}

The research objective is to develop a $2 \mathrm{DOF}$ pedicle screw channel creation simulator. The primary focus is to develop a fully functional vertical probing stage. The vertical stage can be split into two different parts. The first part is to develop the simulation for normal probing situations. The second part is to develop the simulation for the breach scenarios. 
The goal of the simulation is to help surgeons recognizing and avoiding possible breach scenario to occur during real surgery.

\subsection{Summary}

This section discussed the previous works completed on the pedicle screw insertion simulator. The work includes two stages: rotary and vertical. More attention was paid to the rotary stage because it is used in this thesis. In addition, the chapter also discussed the $3 \mathrm{D}$ printed torso and the objective for the next phase of the project. 


\section{Chapter 4}

\section{Linear Actuator Control}

The linear actuator and its control strategy is discussed in this chapter. The linear actuator is designed to simulate probe's vertical movement. There is no control strategy change in the rotary component from Chapter 3. The rotary and vertical stage is coupled together where rotational movement is required in order to start vertical movement. This will be explained further in the chapter. The simulation tasks for the simulator includes normal probing and breach probing.

\subsection{Actuator}

The vertical probing process requires high speed motion with precision control. To simulate the downward motion of the probe, a linear actuator is placed underneath the rotary actuator. The actuator should be able to handle the large downward force created by the surgeon. The typical force created by a surgeon is around $150 \mathrm{~N}$ [33]. Moreover, the surgeon is required to pick up subtle changes in force and dynamic. Quick response is important in 
such situations. In order to recreate this haptics sensation, the actuator should have a fast response and high precision. In the previous stage of the project, an electric actuator was chosen for the project [9]. Electric actuators have many benefits compare to pneumatic and hydraulic actuators. This includes high precision and low maintenance. A DC electric stepper motor actuator was used for the first stage of the development. However, due to the limited bandwidth, the actuator could not respond fast enough using a closed loop approach. Thus, an open loop control strategy was used in the vertical stage to simulate the normal probing process. As for the breach scenario, due to limited current and lack of high precision encoder, the actuators top speed limited to $3 \mathrm{~cm} / \mathrm{s}$. This strongly affects the realism felt by the surgeon. Therefore, it was decided to redesign the linear stage. This is the topic of this chapter.

\subsection{Actuator Replacement}

In replacing the linear actuator, the top speed and response time of the actuator were considered as the top criteria. Based on surgeons' feedback, the new actuator should have an initial thrust of $300 \mathrm{~N}$. This is twice as much force as used by a surgeon in typical operation [33]. The old actuator had a top speed of $3 \mathrm{~cm} / \mathrm{s}$. Because this is significantly slower than what is required for the simulation tasks, the new top speed requirement is set at $15 \mathrm{~cm} / \mathrm{s}$, which is five times higher than before. Pneumatic and hydraulic actuators often have high speed and acceleration, but due to the high cost of the control valves and safety restrictions, a high-power DC electric actuator is chosen for the project. Servo motors are usually linked to high performance, but a modern hybrid stepper motor can also provide high torque and precision with the benefit of low cost. In a normal stepper motor, the motor can only operate in steps that correspond to the number of magnets in 
the motor. In a hybrid stepper motor, the driver can split each step into many micro-steps. Each micro step can be driven separately. This result in high precision and low power loss. As a result, a linear actuator with stepper motor and integrated driver was chosen for the simulator. The selected actuator model is the Bimba OLE-1507-25Q-E2 @ (see Figure 4.1). Using the Bimba STP Controller, the actuator is able to provide an initial thrust of 667 $\mathrm{N}$ and top speed of $0.23 \mathrm{~m} / \mathrm{s}$. At $0.15 \mathrm{~m} / \mathrm{s}$, the actuator has an thrust of $180 \mathrm{~N}$ [55]. After initial tests, it is determined that the selected actuator provides sufficiently fast response time and satisfies the design requirement.

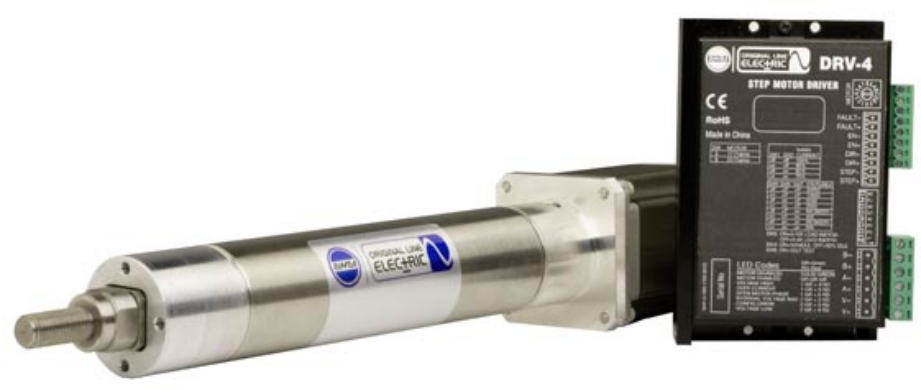

Figure 4.1: Electric actuator with driver[11]

\subsection{Actuator Driven Mechanism}

Based on the design requirement, the actuator needs to run in real time closed loop control. Due to actuator controller's software restriction, the only actuator control mode that can 
be used in real time is the velocity mode. In velocity mode, the actuator is driven by a velocity command. The actuator's controller will calculate the number of micro-steps per second required based on the velocity command. The build in actuator controller will then control the motor to accelerate to the desired speed. The controller frequency is set to $1 \mathrm{kHz}$, which is also the human haptics resolution limit [38]. The data bandwidth of the controller is limited to the 115 thousand baud per second. Each command block uses 32 baud, and there are three essential blocks for the communication. In total, the current controller communication is using $83.5 \%$ of the total bandwidth. Since changing acceleration and deceleration during operation will add two extra command blocks into the communication bus, this can lead to a transmission bottleneck. Therefore, a constant acceleration and deceleration variable is set before the actuator can commence movement. The constant is determined by finding the highest possible acceleration without any mechanical slip in the actuator. Since motor speed is directly related to the input current from the driver, the actuator controller can automatically increase operation current to account for the additional external force and friction applied on the actuator. Due to the fast response time of the driver, a sudden increase in external force should not affect actuator's performance. This is especially important because the simulator is required to handle extra forces during breach scenarios.

\subsection{Normal Probing Control model}

In haptics feedback applications, admittance control is one of the most commonly used control techniques. It uses the external force to manipulate the movement of the surrounding environment or the virtual surface. In this case, the environment is the linear actuator. Under soft or fast response environment, admittance control can provide robust and pre- 
cise performance [56]. Figure 4.2 illustrates the linear actuator control scheme, where $x_{0}$ is the equilibrium position. $\dot{x}_{d}$ is the desired velocity. $\mathrm{x}$ and $\dot{x}$ are the actuator position and velocity. $\mathrm{F}$ is the actuator force. $F_{e x t}$ is the external force. $\dot{\theta}$ is the rotary velocity. The control system has two control loops. The inner loop is contained inside the linear actuator and cannot be reconfigured. It is controlled by the velocity control block. The velocity controller is able to use the velocity feedback from the encoder to achieve desired velocity. At rest, the actuator is able to create a large initial thrust of $667 \mathrm{~N}$ to overcome static friction. Due to the fast acceleration of the actuator, the actuator environment is considered to be soft. Therefore, admittance control is chosen as the outer loop controller. This controller is developed from the PC side using Matlab.

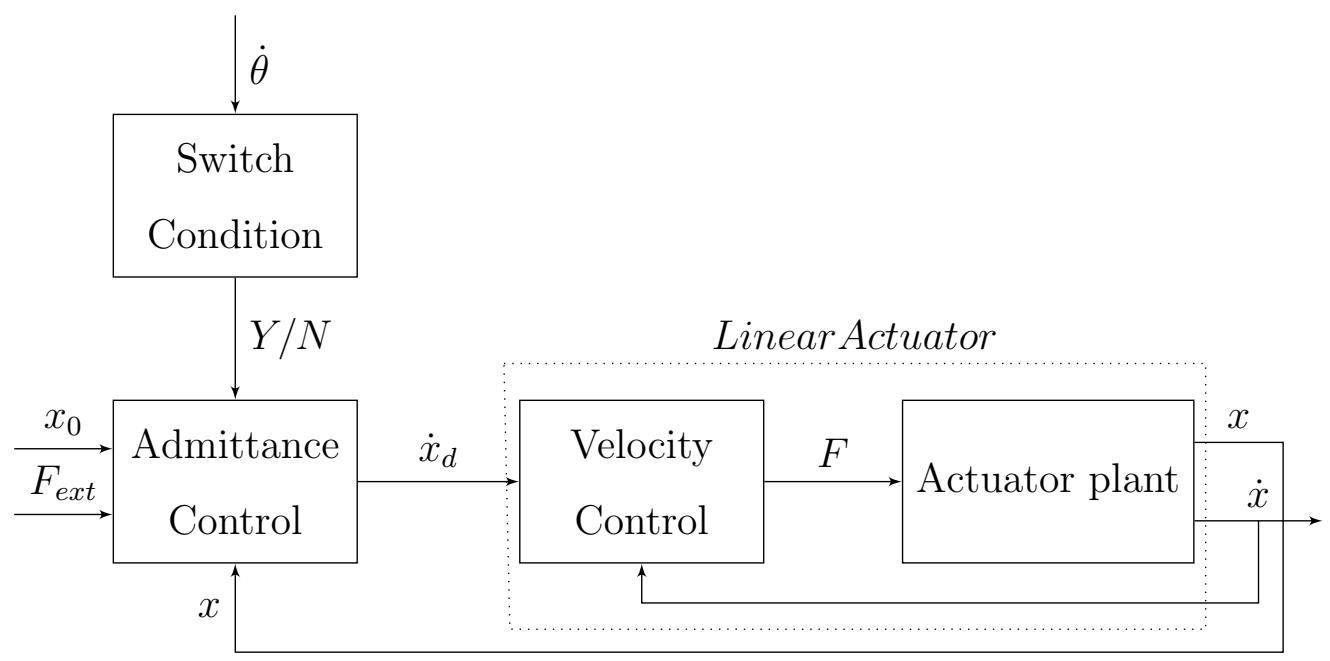

Figure 4.2: Block diagram of the linear actuator control scheme.

When the surgeon is probing inside the pedicle, one important detail is that the surgeon has to start rotating the pedicle before using any vertical force. Therefore, a switch condition is added to the admittance controller. The admittance controller is only active after the rotary velocity is more than 10 degrees per second. 
Inside the admittance controller, a virtual mass spring damper system is used to simulate pedicle insertion dynamics. The behaviour of the actuator is determined by a linear second order system.

$$
M\left(\ddot{x}-\ddot{x}_{0}\right)+D\left(\dot{x}-\dot{x}_{0}\right)+K\left(x-x_{0}\right)=F_{e x t}
$$

where:

$$
\begin{aligned}
& M=\text { Virtual Inertia Coefficient } \\
& D=\text { Virtual Damping Coefficient } \\
& K=\text { Virtual Stiffness Coefficient / Spring Coefficient } \\
& x, \dot{x}, \ddot{x}=\text { Actuator Position, Velocity, and Acceleration } \\
& x_{0}, \dot{x}_{0}, \ddot{x}_{0}=\text { Equilibrium Position, Velocity, and Acceleration } \\
& F_{e x t}=\text { External Force }
\end{aligned}
$$

Because of the high stiffness and damping of the pedicle, the relative small mass of the pedicle is assumed to be zero. The actuator position is also assumed to be same as the desired actuator position. This is due to the fast response of the linear actuator, where the difference between desired position and actuator position is very small. Moreover, the high sample rate and minimum hardware delay indicates the system has relatively fast response. The external force is said to be equal to the control force $\mathrm{F}$ assuming the control force are small. From this, Equation 4.1 can be further simplified to:

$$
D\left(\dot{x}_{d}-\dot{x}_{0}\right)+K\left(x_{d}-x_{0}\right)=F
$$

Assuming a virtual mass spring damper system have zero velocity at the surface of the system, the equilibrium velocity can be assumed to be equal to zero. By using Equation 
4.2, the desired velocity feed into the actuator can be obtained from the following equation:

$$
\dot{x}_{d}=\left(F-K\left(x_{d}-x_{0}\right)\right) / D
$$

Under normal pedicle screw insertion surgery, the probing process can be described as breaking though layers of a lattice. From the haptics perspective, the sensation can be described as a series of strong vibration. This is due to the non uniform density distribution of the pedicle bone. To simulate this effect, the vertical probing length is broken into many small layers, each layer represented by its own mass-spring-damper system. An illustration of the probing process can be seen in Figure 4.3 A). Inside each layer, the actuator's motion will follow Equation 4.3, The spring and damping coefficient is unique to that layer. The haptics sensation is the same as pushing on a spring and damper system. This is shown in Figure 4.3 B). Each layer is assigned with its own depth. The system will switch to a new layer once the probe reaches the end of the previous layer. Each new layer has its own mass spring damper system. The boundary between each system represents the change in property of the bone density. A sudden change in resistance should be felt by the operator. Because each layer's density is different, the property of each section also has to be different from the others. A switching controller is added to change the layer's parameter for each layer. An illustration of each section's resistance is shown in Figure 4.3. 


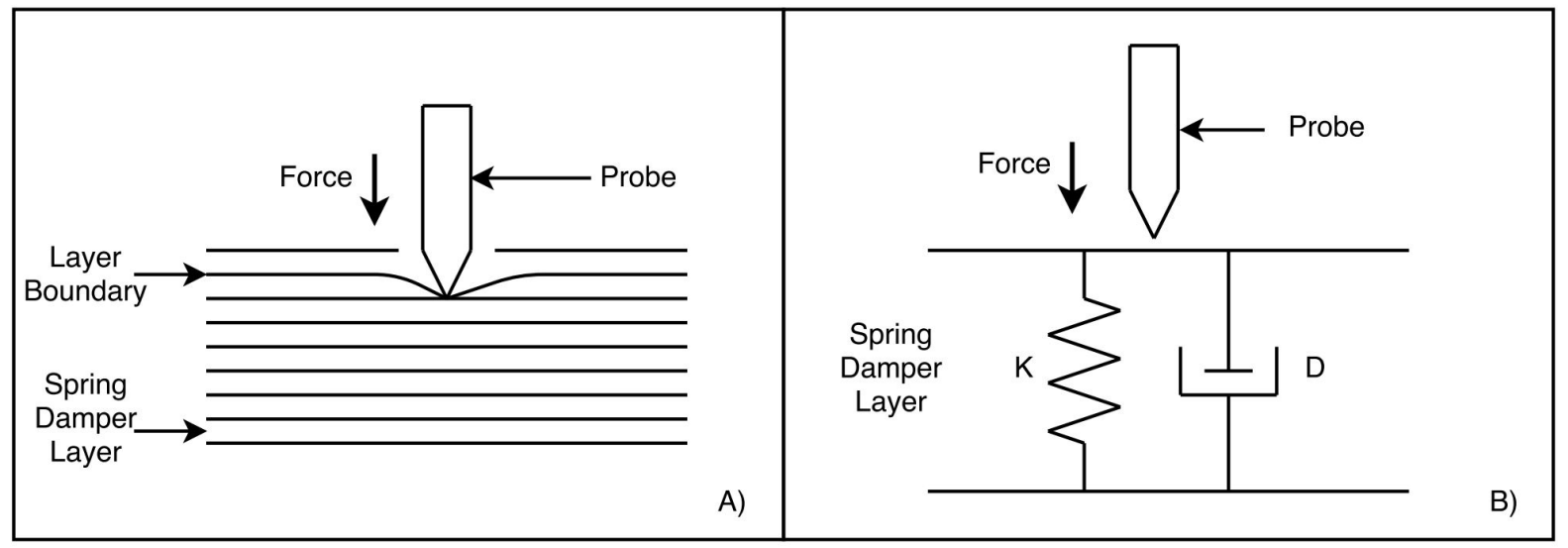

Figure 4.3: Spring damper model of the pedicle bone. A) Probe drilling into the pedicle layer. B) Spring and damper inside each layer

In total, there are four parameters to be tuned in normal probing, they are listed in Table 4.1.

Table 4.1: Normal probing tuning parameters

\begin{tabular}{|l|}
\hline $\begin{array}{l}\text { Normal Vertical Stage } \\
\text { Probing Parameters }\end{array}$ \\
\hline Spring Coefficient $\mathrm{K}$ \\
\hline Damper Coefficient $\mathrm{D}$ \\
\hline Equilibrium Point $\mathrm{x}_{0}$ \\
\hline Equilibrium Interval Scale $\mathrm{L}$ \\
\hline
\end{tabular}

\subsection{Breach Simulation}

Breach simulation is an extension to the normal pedicle probing procedure. In most cases, the breach haptics sensation is different compared to normal probing. The breach simulation procedure can be split into two parts. The first part is before the breach, where the 
probe is experiencing increased resistance. The second part is after the breach occurred, where the resistance is minimum. The controller uses two different sets of parameters to simulate the haptics sensation.

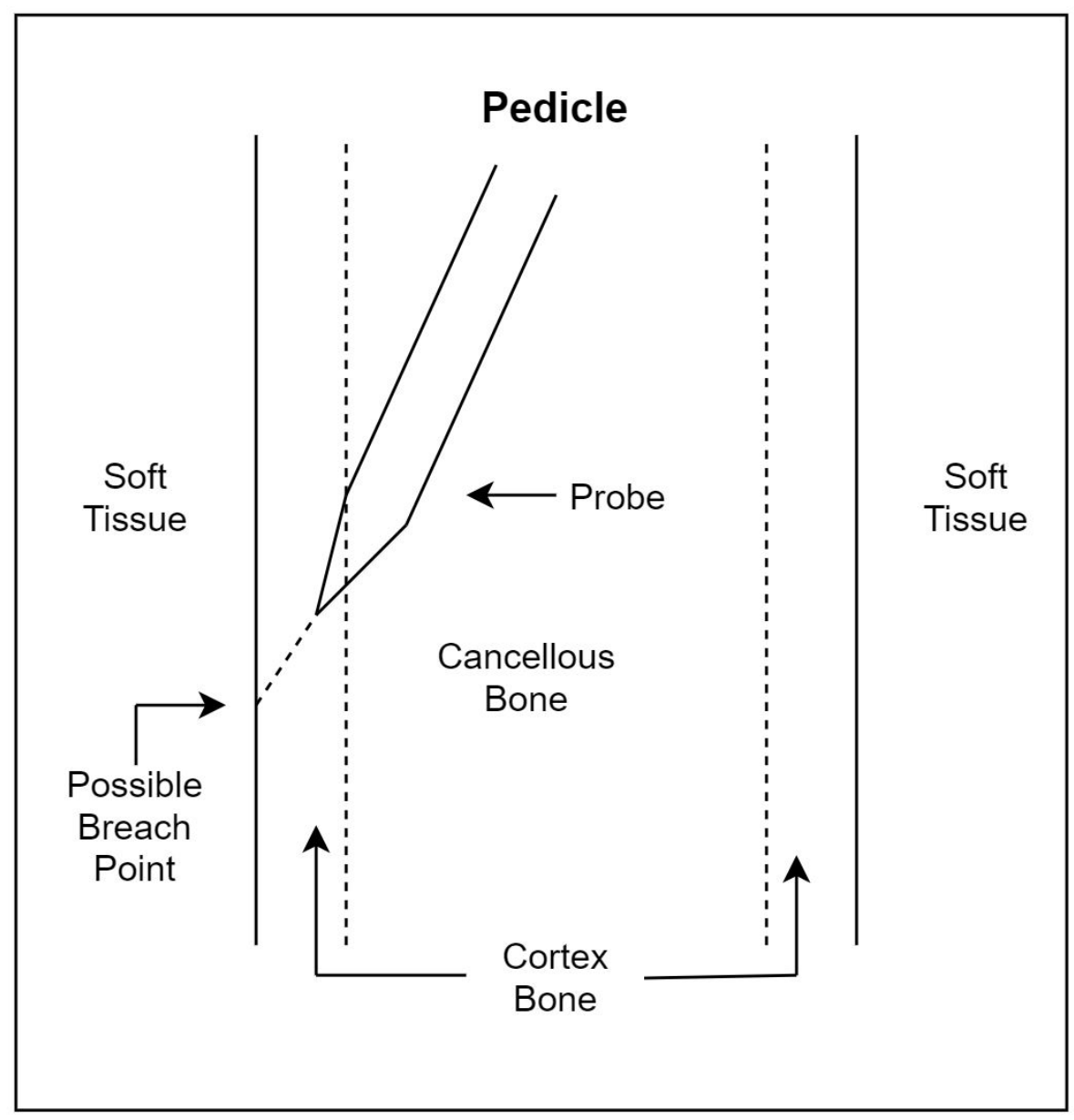

Figure 4.4: Illustration of breach reaching cortex bone of the pedicle

In most surgery conditions, there are obvious warnings before breach occurs. Surgeons have to recognize these warnings in order to avoid breach. The most important warning is the increasing probing resistance. This indicates the probe have reached the outer cortical 
of the pedicle (see Figure 4.4). More often then not, the increase in resistance is due to narrow pedicle size. In some case, the increase in resistance indicates that the probe is placed at the wrong angle and a breach may occur if the surgeon decides to continue probing. An illustration of such event is shown in Figure 4.4. In this situation, if the surgeon does not feel comfortable to continue probing, then she or he can choose to readjust the probe position and then continue probing. An illustration of a typical resistance change is shown in Figure 4.7. If the surgeon is determined to continue probing without stopping, then the surgeon has to increase the navigation force to overcome probing resistance or correcting the probe position. An illustration of a typical resistance change with probe adjustment is shown in Figure 4.5.

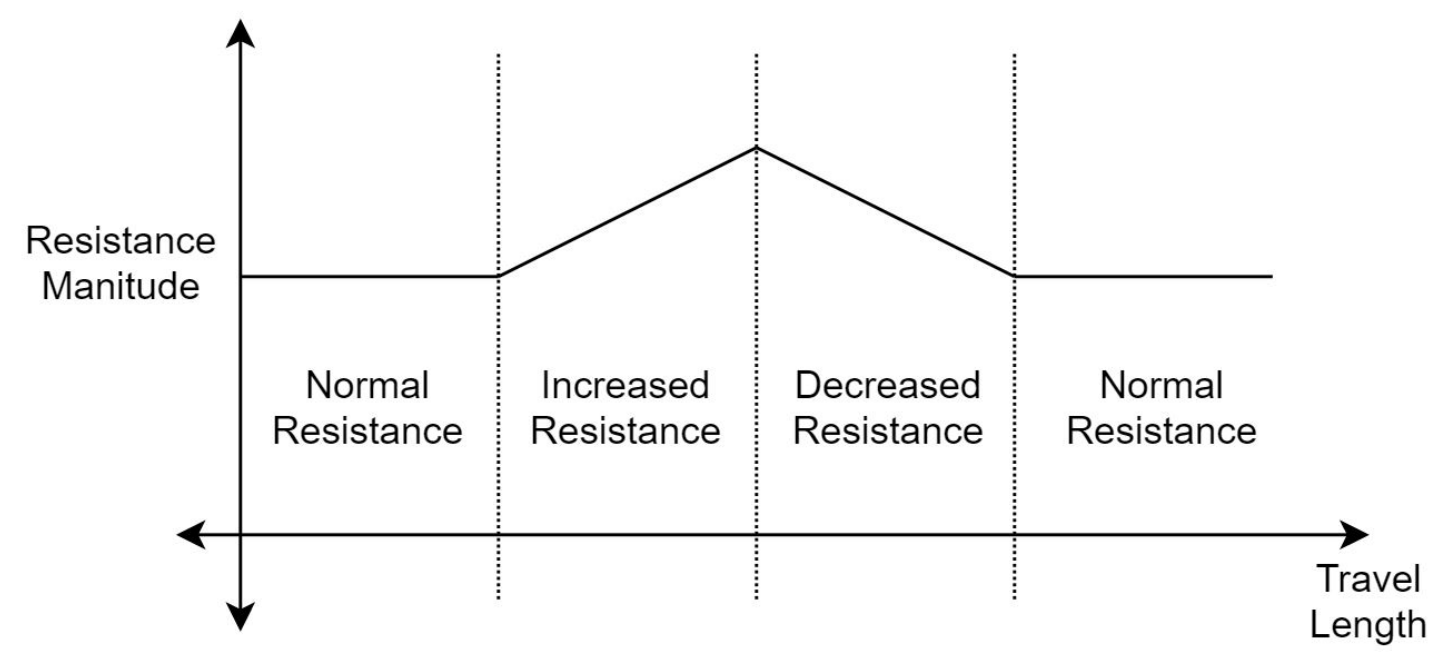

Figure 4.5: Resistance magnitude change with respect to the probe travel length in constant applied force 


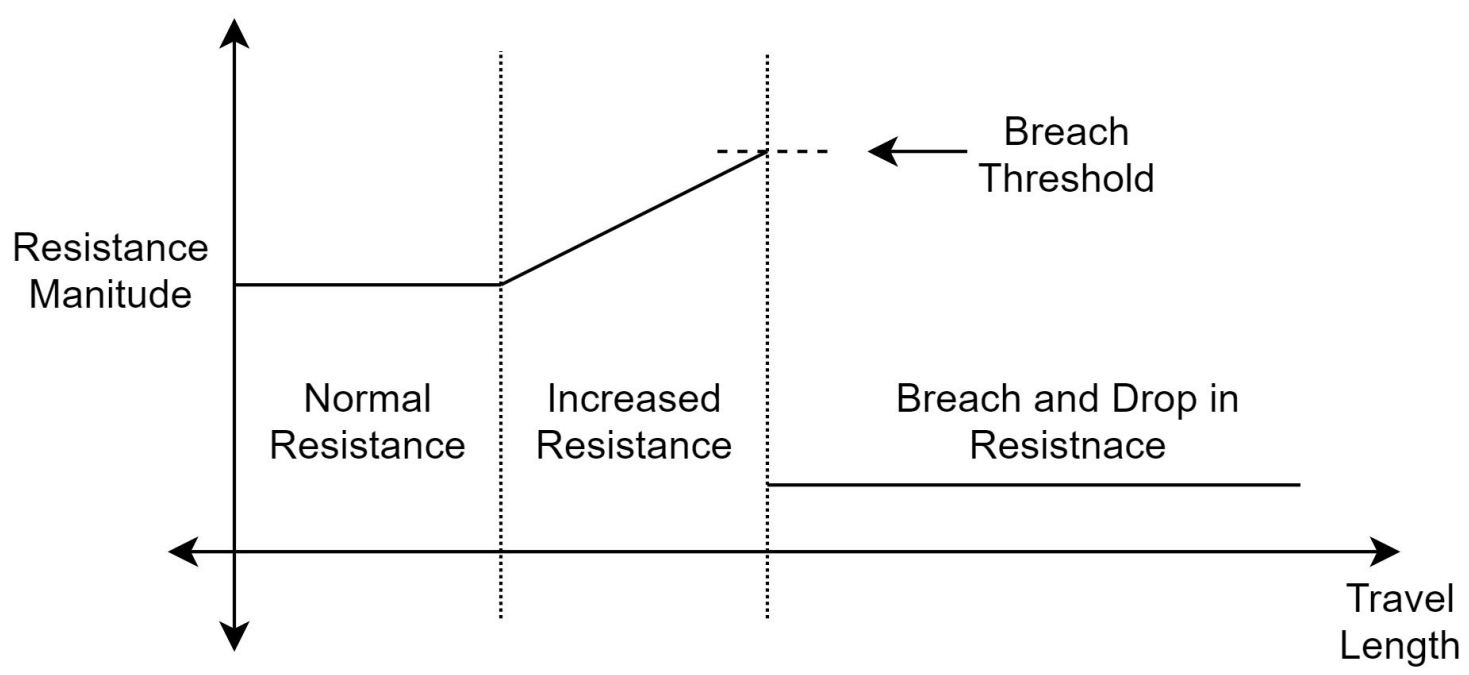

Figure 4.6: Resistance magnitude change during breach with respect to the probe travel length

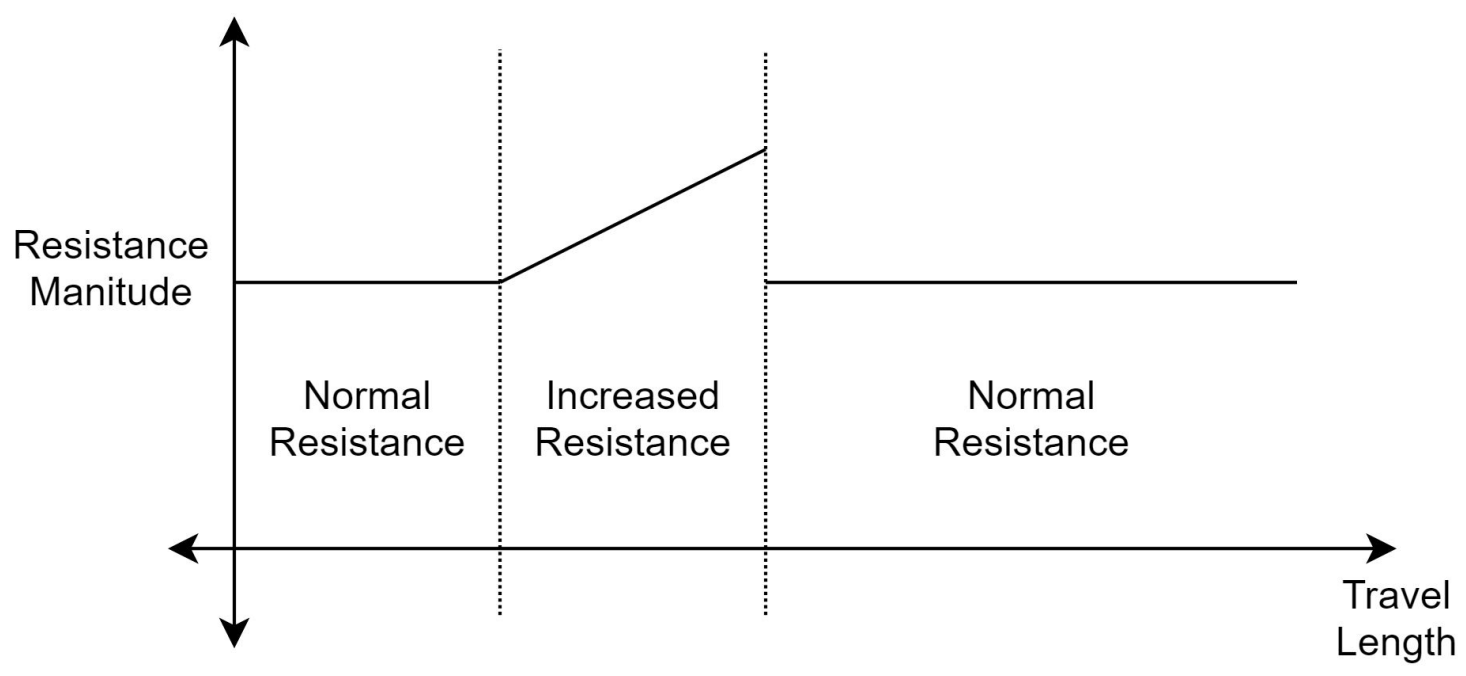

Figure 4.7: Resistance magnitude change in probe adjustment scenario with respect to the probe travel length 
Table 4.2: Breach tuning parameters

\begin{tabular}{|l|}
\hline Breach Parameters \\
\hline Resistance Interval $\mathrm{R}_{\mathrm{i}}$ \\
\hline Resistance Factor $\mathrm{R}_{\mathrm{f}}$ \\
\hline Breach Interval $\mathrm{L}_{\mathrm{b}}$ \\
\hline Breach Damping Coefficient $\mathrm{D}_{\mathrm{b}}$ \\
\hline
\end{tabular}

For the implementation, a reference location is used as a starting point for the increase in probing resistance. A parameter called resistance interval $R_{i}$ is used to model the distance travelled by the probe between the reference location and the actual breach location. When the probe is travelling during this interval, the probing resistance is increased linearly. A scaling parameter, resistance factor $R_{f}$, is used as the slope of the change. The spring and damping coefficient from Equation 4.3 will increase based on the resistance factor and probe location. An illustration of the change is shown in Figure 4.6 in the increase resistance section. If the probe reaches past the pedicle wall, breach will begin. The breach resistance is decreased significant. The breach point is identified as breach threshold in the simulation. This is the largest possible force exerted on the probe before breach. The tuning parameter for the scenario is shown in Table 4.2. The detail will be explained later in the section.

If the surgeon is able to avoid breach by correcting the direction of the probe, the probing resistance is than decreased back into normal amplitude (see Figure 4.5). For the simulation, if the operator decides to pause probing during the increasing resistance interval, then the simulator will assume the probe's path is been corrected by the surgeon (shown in Figure 4.7). No breach will occur during the remainder of the trial.

The most common breach scenario is breaching into surrounding soft tissues. This haptics sensation is much softer than probing inside the pedicle. In order to adapt to this 
change, the vertical direction admittance controller's parameters are readjusted for the simulation. If a breach has occurred, then the resistance of simulator will drop dramatically, which results in sudden drop in the vertical resistance. This is shown is Figure 4.6. The low breach resistance is accomplished by setting the stiffness coefficient to zero, and the damping coefficient to a much smaller number. The desired velocity feed into the actuator from Equation 4.3 is now simplified to the following equation:

$$
\dot{x}_{d}=F / D_{b}
$$

where $D_{b}$ is the breach damping coefficient.

During breach, the soft tissue and body fluid can reduce rotational resistance, in most cases, the rotational resistance is close to zero. A rotational switch is implemented to shut down the rotational motor after breach has occurred. Since the rotational motor is a servo motor, shutting down is motor does not lock the rotation. Therefore, the rotational resistance is close to zero.

In total, there are two parameters that needed to be tuned during breach: breach interval $L_{b}$ and breach damping coefficient $D_{b}$. Breach interval is the total allowed travel length of the breach and the breach damping coefficient is used to control the speed of the breach. The tuning of breach simulation is discussed in the next chapter. 


\subsection{Linear Control System}

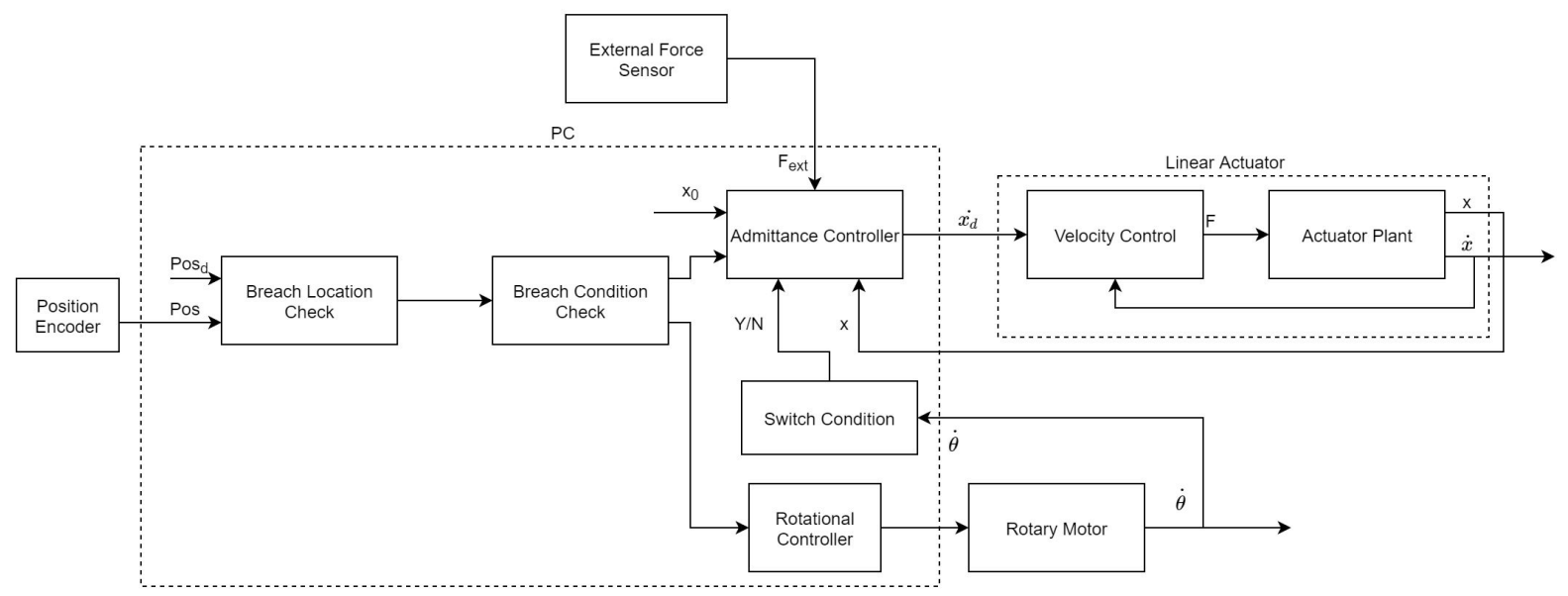

Figure 4.8: Block diagram of the vertical component

Figure 4.8 illustrate the complete block diagram of the linear actuator. In order to simulate real surgical procedure, 8 unique parameters have to be tuned in the linear stage. The parameter are shown in Table 4.3. The detail of the tuning process can be found in the next chapter.

Table 4.3: Linear stage tuning parameters

\begin{tabular}{|l|l|}
\hline $\begin{array}{l}\text { Normal Probing } \\
\text { Parameters }\end{array}$ & Breach Parameters \\
\hline Spring Coefficient $\mathrm{K}$ & Resistance Interval $\mathrm{R}_{\mathrm{i}}$ \\
\hline Damper Coefficient $\mathrm{D}$ & Resistance Factor $\mathrm{R}_{\mathrm{f}}$ \\
\hline Equilibrium Point $\mathrm{X}_{0}$ & Breach Interval $\mathrm{L}_{\mathrm{b}}$ \\
\hline Equilibrium Interval Scale $\mathrm{L}$ & Breach Damping Coefficient $\mathrm{D}_{\mathrm{b}}$ \\
\hline
\end{tabular}




\subsection{Summary}

The vertical stage implementation was discussed in this chapter. The previous design flaw and replacement process was discussed. The control strategy was explained. The control strategy includes two main components: normal probing and breach. Each is detailed in this chapter. 


\section{Chapter 5}

\section{Clinical Testing}

To create a realistic simulation, a simulator tuning session was conducted by Dr. Zeller and Dr. O'Shea from Toronto SickKids Hospital. The detail of the tuning session is ex-

plained in this chapter. In order to evaluate the effectiveness of the tuning, a clinical study is conducted with help of 10 other surgeons from SickKids Hospital. The experimental procedure of the study is explained in this chapter. Lastly, the research questions and null hypotheses are outlined in this chapter.

\section{$5.1 \quad$ Simulator Tuning}

To create a realistic simulation, a simulator tuning session is conducted with help of Dr. Zeller and Dr. O'Shea. They are both expert surgeons with at least 3 year of scoliosis surgery experience. In particular, Dr. Zeller is a world class spine surgeon with over 25 years of experience [57]. And Dr. O'Shea has more than 5 years of experience [58]. The end goal of the tuning session is to recreate surgical scenarios that can be used to 
train and evaluate surgeons. In this thesis, 10 other surgeons performed these simulation scenarios during a clinical study, the results from the study are used to answer research questions outlined in this chapter. The tuning session includes two parts: informal tuning session and formal tuning session. The informal tuning session was conducted to help surgeons familiarize with the simulator. The surgeon is asked to experiment with the tuning parameters. The tuning parameters from the informal tuning session are used as a starting point for the formal tuning session. The formal tuning session can be split into three different parts. The first session is conducted to recreate simulation with normal probing condition. The second session is used to generate simulations in breach situations. The third session combines and modifies the previous two sessions to simulate complex scenarios that can occur during the surgery.

It is important to note that expert's tuning parameters may not reflect the real surgical sensation. Further testing with other experts is required to verify the realism of the scenarios. This study is preliminarily focused on the feasibility of the simulator. It is also used to evaluate the performance difference between surgeons with various experience level.

\subsubsection{First Session}

In the first session, each of the two surgeons was asked to tune the simulator parameters under a normal probing process. The list of parameters can be found in Table 5.1. The surgeon must assume that the probe is perfectly positioned inside the pedicle and the pedicle bone composition is uniform. The simulation should simulate the most common scenario for normal pedicle probing. Before the tuning session begins, the parameters were reset to values recorded from the previous informal tuning session as a starting point. The surgeon was then asked to tune each parameter twice. Under each parameter tuning, the 
target parameter is initialized to two values where each is 1.5 times larger or smaller than the informal session's tuning value. All other parameters must remain constant during the tuning process. The surgeon was asked to adjust the parameter based on the haptics feedback. The tuning was completed using staircase procedure. After the tuning procedure for the variable was finished, the average of the two tuning numbers was used as the new tuning parameter. The simulator was then reset back to the old parameters in order to tune the next parameter. This can reduce error from tuning bias. The process was repeated until all parameters are tuned. After the tuning was completed and the new parameter values are set in the simulator, the surgeon was asked to refine the haptics feel of the simulator. Due to surgeon's familiarity with the simulator, the surgeon was asked to adjust all the parameters together. The surgeon can only stop tuning if he feels the simulation is realistic.

Table 5.1: Vertical stage and normal linear stage tuning parameters

\begin{tabular}{|l|l|}
\hline $\begin{array}{l}\text { Linear Stage Probing } \\
\text { Parameters }\end{array}$ & $\begin{array}{l}\text { Normal Vertical Stage } \\
\text { Probing Parameters }\end{array}$ \\
\hline Detent interval $\theta_{\mathrm{i}}$ & Spring Coefficient $\mathrm{K}$ \\
\hline Detent width $\theta_{\mathrm{w}}$ & Damper Coefficient $\mathrm{D}$ \\
\hline Detent magnitude $\mathrm{k}_{\mathrm{p}}$ & Equilibrium Point $\mathrm{x}_{0}$ \\
\hline Viscous friction coefficient $\mathrm{k}_{\mathrm{d}}$ & Equilibrium Interval Scale $\mathrm{L}$ \\
\hline
\end{tabular}

\subsubsection{Second Session}

Next, the surgeons were asked to tune the simulator under breach conditions. Breach can occur under many different scenarios. To provide a consistent result, the surgeons were asked to perform the calibration that is closely related to the most common breach scenario: lateral breach into nearby soft tissues. In this situation, the probe will experience a linear increase in resistance before breaching. After the probe reaches the breach threshold, 
the surgeon will feel a sudden drop in resistance. The breach resistance will hold steady until the end of the trial. The start of the breach simulation was set at $5 \mathrm{~cm}$ below the trial starting position. Similar to the first session, each breach parameter was adjusted independently with all other parameters remaining the same. A final adjustment was performed to reduce any unwanted haptics feedback. The breach tuning parameters for this session are listed in Table 5.2. This lateral breach is used as an experimental scenario for the actual clinical study. It also serves as a base for other breach scenario tuning such as the in-out-in probing.

Table 5.2: Breach tuning parameters

\begin{tabular}{|l|}
\hline Breach Parameters \\
\hline Resistance Interval $\mathrm{R}_{\mathrm{i}}$ \\
\hline Resistance Factor $\mathrm{R}_{\mathrm{f}}$ \\
\hline Breach Interval $\mathrm{L}_{\mathrm{b}}$ \\
\hline Breach Damping Coefficient $\mathrm{D}_{\mathrm{b}}$ \\
\hline
\end{tabular}

\subsubsection{Third Session}

During the last session, the surgeons were asked to tune the simulator to more complex conditions based on the previous tuning. Many scenarios were discussed and tuned. The two surgeons were asked to try and rank all the scenarios. The most realistic scenarios were picked for the clinical study. In the end, four scenarios were included in the clinical study. These scenarios are hard bone probing, soft bone probing, lateral breach, and inout-in probing. The illustration of these scenarios is shown in Figure 5.1. The first two scenarios(hard probing and soft probing) are variation of normal probing, the simulation is achieved by scaling the vertical tuning parameters according to the bone density. Each scenario has its own specific spring damper system. In the end, the surgeons were asked to perform the soft, normal, and hard probing side by side, this is to confirm that there 
is enough difference between the three scenarios. The lateral breach scenario developed in the previous session, there is no modification during this session. The in-out-in probing is an extension to lateral breach. In this case, after the probe breaches into the soft tissue. Due to the curvature of the pedicle, the probe can be navigated back inside the pedicle (shown in Figure 5.1). This technique can be used if the patient's pedicle is too small to fit the probe [33]. The tuning procedure for first half of this scenario is the same as lateral breach tuning, where the probe breach into soft tissue. Due to the probe's travel path, where the probe channel back into the pedicle, the latter part of this scenario is the repeat of the former part but in reverse order. 


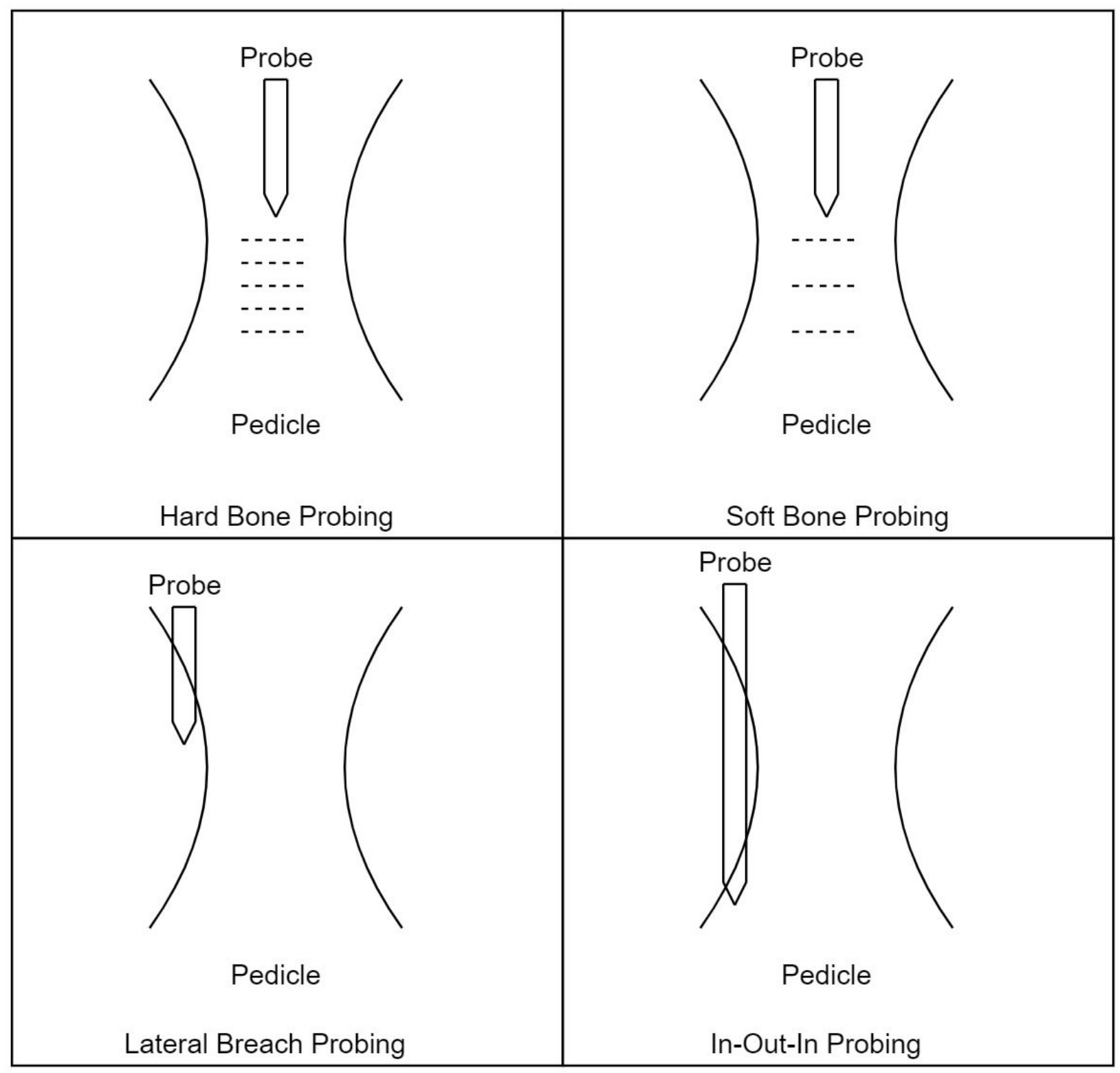

Figure 5.1: Four simulation scenarios developed by the surgeons 


\subsection{Participants}

In total, 10 surgeons have conducted the clinical study. All of them are orthopedic surgeons from Toronto SickKids Hospital. The surgeons are composed of 2 senior surgeons, 4 fellows, and 4 residents. The most experienced surgeon has 6 years of experience in pedicle screw insertion surgery and the residents have just started learning about the surgery. The precise experience level of each surgeon is described in the next chapter. Because the study requires the surgeon to have no previous knowledge of the simulation scenarios, the two expert surgeons, Dr. Zeller and Dr. O'Shea, who helped tuning the simulator did not participate in the study.

\subsection{Experimental Procedure}

\subsubsection{Physical Set-up}

First, the simulator is placed in an open space. The torso body is then positioned on top of the simulator. The height of the actuator is adjusted to provide enough space between the probe and the fixed torso. Because each participant requires different operating table height, an adjustable stand is provided to the participant. The participant should hold the simulator probe at highest operating height, which is used as a reference point for stand adjustment. The simulator workstation is placed so that the participant cannot see the computer monitor. This is to ensure the participant is blind to the testing result. To reduce environment distractions, a pair of noise cancelling headphones is provided to each participant. 


\subsubsection{Training Session}

In order to help participants understand the simulator and the study, each participant is asked to go though a quick training session. During this session, the idea and purpose of the surgical simulator is introduced to the surgeon. The haptics methodology and simulator mechanics are explained to the surgeon. The rotary and vertical stage of the simulator is presented to the participant. Lastly, the participant is asked to test the simulator using normal probing parameters. They are encouraged to ask questions during the trial test. The session will end once the participant is comfortable to start the formal study.

\subsubsection{Experimental Protocol}

Participants were asked to complete a set of 12 trials on the surgical simulator. The trials can be broken down into four different scenarios with each scenario performed three times. The order of the trials was randomized. The scenarios, as discussed in the previous section, are listed below:

- Hard normal insertion

- Soft normal insertion

- Lateral breach into soft tissues.

- In-out-in probing with lateral Breach

For each trial, the participant's objective is to create a screw channel using the probe in a single motion. Ideally, the participant should reach the designated probe stop point without any motion pause. This includes breach scenarios. 
For the study, the participants are blind to the number of scenarios and the trial order. However, the participant is informed that the test scenarios are free hand pedicle screw insertion simulation that includes potential breach simulation. After each trial is completed, the participant is asked whether they experienced a breach during the trial. If the answer is yes, then a additional question is asked to confirm the location of the breach. The simulation is completed when the participant completes all 12 trials.

\subsubsection{Survey Questionnaire}

At the end of the study, each participant is asked to fill out a questionnaire regarding the realism of the simulator. Each question is evaluated using a Likert scale.

\begin{tabular}{|c|c|c|c|c|}
\hline Strongly Disagree & Disagree & Undecided & Agree & Strongly Agree \\
1 & 2 & 3 & 4 & 5 \\
\hline
\end{tabular}

The survey questions:

1. The simulator produced realistic haptics sensations during normal probe channelling.

2. The haptics sensation associated with bone breach was simulated realistically.

3. The simulator could potentially be a useful training tool for teaching normal pedicle screw insertion surgery.

4. The simulator could potentially be a useful training tool for bone breach prevention. 


\subsection{Research Question}

The purpose of the clinical study is to answer 4 research questions. Each research question includes a null hypothesis. The study result is used to find if the null hypothesis is rejected.

1. Can experience help the surgeon improve correct breach recognition rate?

$H_{0}$ : The surgeon's experience background has no effect on breach recognition rate.

One of the main complication of scoliosis surgery is the breach recognition. The breach recognition rate in the study is defined as the rate of correct recognition for lateral breach and in-out-in breach scenarios. Effective breach avoidance can help eliminate surgical risks. It is important to evaluate whether experience can help surgeon in better recognizing breach.

2. Can experience help the surgeon improve overall correct recognition rate?

$H_{0}$ : The surgeon's experience background has no effect on overall wrong recognition rate.

Although not as important as breach recognition, if a surgeon has any false recognition, the surgery can become more complex. It is important to evaluation whether experience can provide any advantage for overall recognition rate.

3. Is there any performance difference between surgeons with different experience levels? $H_{0}$ : The Surgeon's experience has no effect on the following measured data: operation time, breach time, velocity variance, mean force and force variance.

Although simulation performance cannot simply correlate to real surgical performance, it is believed that the simulator can help differentiate between participants skill levels. Thus, it would be beneficial to develop performance metrics that are 
related to participants skill level. In this case, the performance metrics are completion time, breach time, velocity variance, vertical force, and vertical force variance. The force, time, position data can be directly retrieved from the simulator. Other parameters are calculated after the test. Velocity is not included in the performance metric because the travel length is fixed, and operation time is the same as the probe velocity. It is important to examine the velocity variance and vertical force variance because stability is one of the most important skill for surgeons, and variance is an important part in judging stability. Statistical analysis is conducted, and the results will be used to evaluate the efficiency of these performance metrics. The rotary stage performance metric will not be discussed because surgeons use varies different technique based on different rotational movement. There is not enough data to evaluate each surgical technique and distinguish between different techniques.

4. Can the simulation trials become a learning tool for the simulation tasks?

$H_{0}$ : Simulation trial cannot be a learning tool for the simulation tasks.

The validation of the simulation trials can be important on evaluating the procedure design. It also can be used as an effective way to evaluate whether the surgical simulator is an effective training tool. The breach scenario is examined in the research question because this is main training scenario for the simulator. The breach velocity, breach velocity variance, breach force variance, and breach mean force data over the trial time will be used to complete the study. These data can provide an idea of how each surgeon's progression during breach scenario. It can show if the surgeon demonstrated any performance improvement from the first trial to the third trial. 


\subsection{Summary}

This chapter outlines the basics of the clinical study. First, the tuning session conducted by two expert surgeons is presented. Four simulation scenarios were developed during the tuning session. Next, the experimental procedure and participants are discussed. Lastly, the research questions and null hypothesis are explained in this chapter. 


\section{Chapter 6}

\section{Discussion}

This chapter is mainly dedicated to the analysis of the collected data from the clinical study. The four research questions from the previous chapter will be discussed. The null hypothesis is verified with different statistical analysis. The survey questionnaire result is also analysed in this chapter.

\subsection{Research Question No. 1}

Can experience help the surgeon improve correct breach recognition rate?

$H_{0}$ : The surgeon's experience background has no effect on breach recognition rate. 
Table 6.1: Incorrect identification results from lateral breach and in-out-in breach.

\begin{tabular}{|r|r|}
\hline $\begin{array}{l}\text { Surgeon } \\
\text { Experience (years) }\end{array}$ & $\begin{array}{l}\text { \# of Wrong Breach Identifications / } \\
\text { Total Breach Trials }\end{array}$ \\
\hline 6 & \\
\hline 3 & $2 / 6$ \\
\hline 1 & $1 / 6$ \\
\hline 1 & $0 / 6$ \\
\hline 0.5 & $1 / 6$ \\
\hline 0.33 & $0 / 6$ \\
\hline 0.1 & $0 / 6$ \\
\hline 0 & $0 / 6$ \\
\hline 0 & $3 / 6$ \\
\hline 0 & $1 / 6$ \\
\hline &
\end{tabular}

The summary of the study results on number of incorrect breach identifications is shown in Table 6.1. The participants are 10 surgeons from SickKids Hospital, they do not include the 2 expert tuning surgeons. The wrong breach identification number is retrieved from the incorrect breach recognition from lateral breach and in-out-in breach. In total, the surgeons performed 6 breach trials. The average correct breach identification rate for the 10 surgeons is $85 \%$. In order to analysis the results, a linear regression t-test is conducted on number of wrong breach identification results with respect to the surgeon's experience. The regression test uses a regression line to estimate the relationship between the independent variable and dependent variable [59]. The regression line equation of the test can be stated as following:

$$
Y=\beta_{0}+\beta_{1} x
$$


where:

$$
\begin{aligned}
& x=\text { Years of Experience } \\
& Y=\text { Number of Wrong Identification } \\
& \beta_{0}=\text { Constant } \\
& \beta_{1}=\text { Regression Coefficient }
\end{aligned}
$$

$\beta_{0}$ and $\beta_{1}$ are calculated using least square estimates. They can be retrieved using the following formula:

$$
\begin{gathered}
\beta_{0}=\bar{y}-\beta_{1} \bar{x} \\
\beta_{1}=\frac{\sum_{i=1}^{n}\left(x_{i}-\bar{x}\right)\left(y_{i}-\bar{y}\right)}{\sum_{i=1}^{n}\left(x_{i}-\bar{x}\right)^{2}}
\end{gathered}
$$

where $x_{i}$ and $y_{i}$ are the observed independent and dependent value of i-th surgeon. $\bar{x}$ and $\bar{y}$ are the mean of the independent and dependent variable. $\mathrm{n}$ denotes the number of data point in the set.

The standard error of the slope is calculated using the following formula:

$$
\begin{gathered}
S E\left(\beta_{0}\right)=\sqrt{\frac{\sum_{i=1}^{n}\left(y_{i}-\hat{y}_{i}\right)^{2}}{n-2}\left(\frac{1}{n}+\frac{\bar{x}^{2}}{\sum_{i=1}^{n}\left(x_{i}-\bar{x}\right)^{2}}\right)} \\
S E\left(\beta_{1}\right)=\sqrt{\frac{\sum_{i=1}^{n}\left(y_{i}-\hat{y}_{i}\right)^{2} /(n-2)}{\sum_{i=1}^{n}\left(x_{i}-\bar{x}\right)^{2}}}
\end{gathered}
$$

where SE is the standard error, and $\hat{y}_{i}$ is the estimated value of $\mathrm{y}$ at variable $\mathrm{i}$. It can be found by the following equation:

$$
\hat{y}_{i}=\beta_{0}+\beta_{1} x_{i}
$$


The t statistic can be calculated using the following equation:

$$
t_{m}=\beta_{m} / S E_{m}
$$

where $t_{m}$ is the $t$ statistic and $\mathrm{m}$ is related to $\mathrm{m}$-th order coefficient in the regression line equation.

The p-value or calculated probability is the conditional probability, given the null hypothesis is true, of obtaining the observed result or more extreme results [60]. If the p-value is smaller than 0.05 , then the result is determined to be statistically significant, which means the null hypothesis is rejected. The p-value is calculated using the formula below:

$$
p=2 * P\left(T_{n-2} \geqslant t\right)
$$

where $\mathrm{p}$ is the $p$-value, $\mathrm{P}$ is the probability of the condition, and $T_{n-2}$ is a $\mathrm{t}$ distributed random variable with n-2 degree of freedom.

By following the standard procedure of regression analysis, a $p$-value is obtained from the test. The results of the test is shown in Table 6.4. It was found that the $p$-value number of wrong breach identification test is 0.3890 . In this test, the $p$-value is significantly larger than the typical significant level of 0.05 . Therefore, the null hypothesis is not rejected. Based on the results, surgeon's experience has no effect on the number of wrong breach identifications.

Table 6.2: Regression analysis results for surgeon's experience vs number of wrong breach identification.

\begin{tabular}{|l|l|l|l|l|}
\hline Predictor & Coefficient $\left(\beta_{\mathrm{m}}\right)$ & SE Coefficient $\left(\mathrm{SE}\left(\beta_{\mathrm{m}}\right)\right)$ & $\mathrm{T}\left(\mathrm{t}_{\mathrm{m}}\right)$ & $\mathrm{P}\left(\mathrm{p}_{\mathrm{m}}\right)$ \\
\hline Constant $(\mathrm{m}=1)$ & 0.7105 & 0.3796 & 1.8717 & 0.0981 \\
\hline $\begin{array}{l}\text { Years of } \\
\text { Experience }(\mathrm{m}=2)\end{array}$ & 0.1588 & 0.1744 & 0.9106 & 0.3890 \\
\hline
\end{tabular}


This result can be related to the study conducted by Samdani et al [30], where the study has shown that there is no statistical significance between experienced and inexperienced surgeons in non medial breach rate. Further study and more data would potentially help understanding why such phenomenon exist.

\subsection{Research Question No. 2}

Can experience help the surgeon improve overall correct recognition rate?

$H_{0}$ : The surgeon's experience background has no effect on overall wrong recognition rate.

Table 6.3: Total number of incorrect identification results

\begin{tabular}{|r|l|}
\hline $\begin{array}{l}\text { Surgeon } \\
\text { Experience (years) }\end{array}$ & $\begin{array}{l}\text { Total \# of Wrong } \\
\text { Identifications / Total Trials }\end{array}$ \\
\hline 6 & $3 / 12$ \\
\hline 3 & $3 / 12$ \\
\hline 1 & $2 / 12$ \\
\hline 1 & $1 / 12$ \\
\hline 0.5 & $3 / 12$ \\
\hline 0.33 & $0 / 12$ \\
\hline 0.1 & $0 / 12$ \\
\hline 0 & $5 / 12$ \\
\hline 0 & $2 / 12$ \\
\hline 0 & $4 / 12$ \\
\hline
\end{tabular}

The summary of the study results on number of incorrect identifications is shown in 
Table 6.1. The number of wrong identifications is retrieved from all four scenarios, this includes the wrong breach identifications. In total, the surgeons performed 12 trials. The average correct identification rate for the 10 surgeons is $81.8 \%$. Similar to research question 1 , a linear regression t-test is conducted on number of wrong identification results with respect to the surgeon's experience. The results are shown in Table 6.4. It was found that the $p$-value for total number of wrong identification test is 0.6920 , which is not statistically significant. Therefore, the null hypothesis is not rejected. It is interesting to note that the p-value is higher in wrong breach identification test than the wrong breach identification test. This shows that surgeon's experience level has no impact on wrong identifications in non-breach scenarios.

Table 6.4: Regression analysis results for surgeons experience vs total number of wrong identification.

\begin{tabular}{|l|l|l|l|l|}
\hline Predictor & Coefficient $\left(\beta_{\mathrm{m}}\right)$ & SE Coefficient $\left(\mathrm{SE}\left(\beta_{\mathrm{m}}\right)\right)$ & $\mathrm{T}\left(\mathrm{t}_{\mathrm{m}}\right)$ & $\mathrm{P}\left(\mathrm{p}_{\mathrm{m}}\right)$ \\
\hline Constant $(\mathrm{m}=1)$ & 2.1537 & 0.6494 & 3.3164 & 0.0106 \\
\hline $\begin{array}{l}\text { Years of } \\
\text { Experience }(\mathrm{m}=2)\end{array}$ & 0.1225 & 0.2983 & 0.4107 & 0.6920 \\
\hline
\end{tabular}

\subsection{Research Question No. 3}

\section{Is there any performance difference between surgeons with different experience levels?}

$H_{0}$ : Surgeon's experience has no effect on the measured data such as operation time, breach time, velocity variance, mean force and force variance. 


\subsubsection{Robust Regression Analysis}

In order to evaluate the difference in the surgeon's performance with different experience level, the collected data was examined and processed to retrieve numerical values such as operation time, velocity variance, mean force, and force variance. The probe velocity is ignored because it is the same as operation time divided by probe travel length, where the probe travel length is constant. First, each scenario was examined separately with regression analysis. In order to account for the variation between trials, the mean of each surgeon's three trials results was taken. Because there is no sudden motion change in the soft probing and hard probing scenario, the complete duration of the trial was examined. Figure 6.1 and Figure 6.2 illustrates the results of four sets of collected data for soft probing and hard probing scenario. For the breach scenario, due to the short length of normal probing travel in breach scenarios, only the breach section of the trial was examined. The result is shown in Figure 6.3. The in-out-in scenario was omitted because the constant change in probe resistance and short stable travel distance, which can produce large error for this analysis. In all three figures, the following are shown in clockwise order: 1) Operation time graph 2) Velocity variance graph 3) Force variance graph 4) Mean force graph. The $\mathrm{x}$-axis is arranged with 1 being the most experienced surgeon and 10 being least experienced surgeon. The experience level can be found in Table 6.1. 

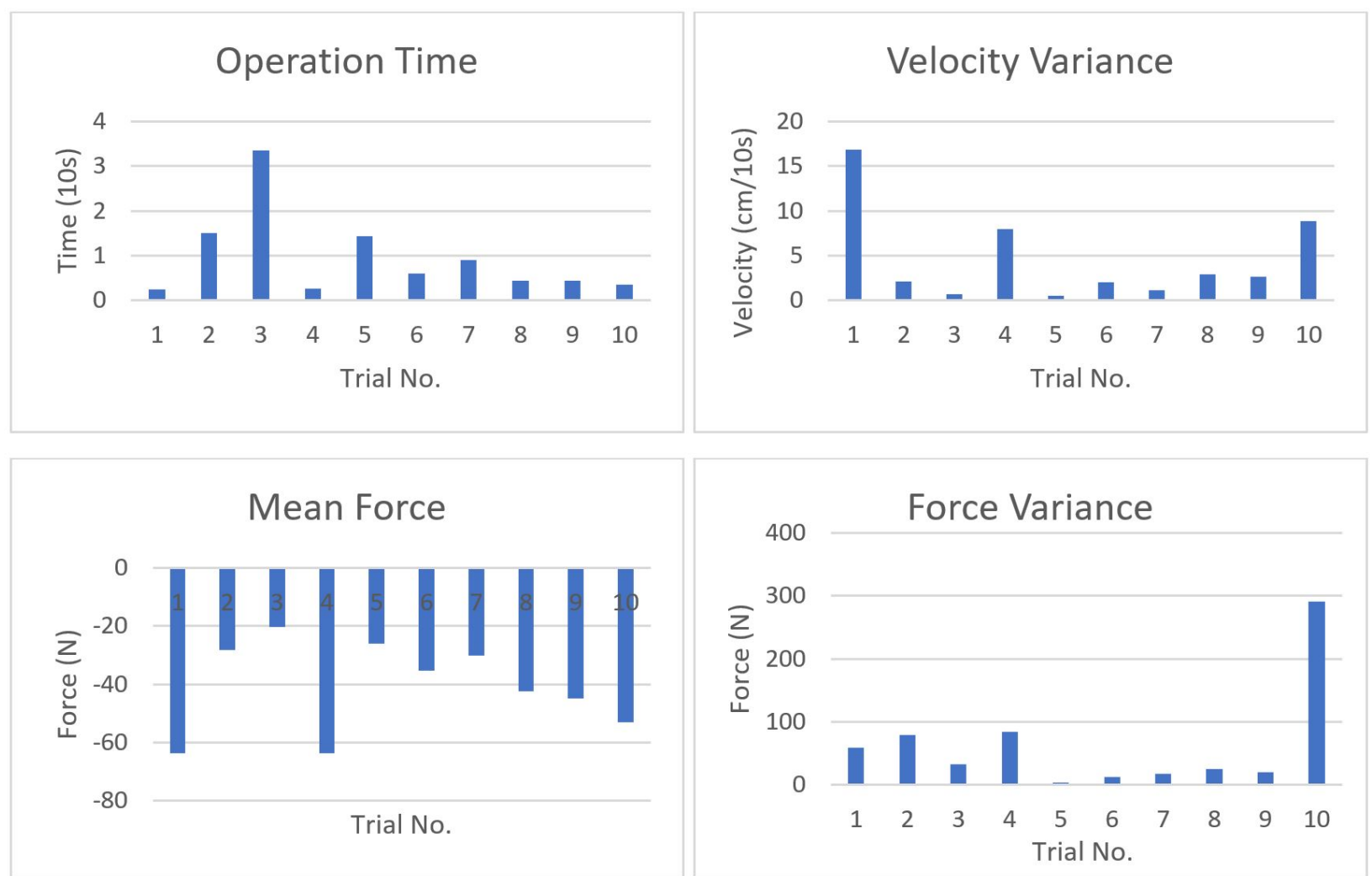

Figure 6.1: Soft probing test results. 

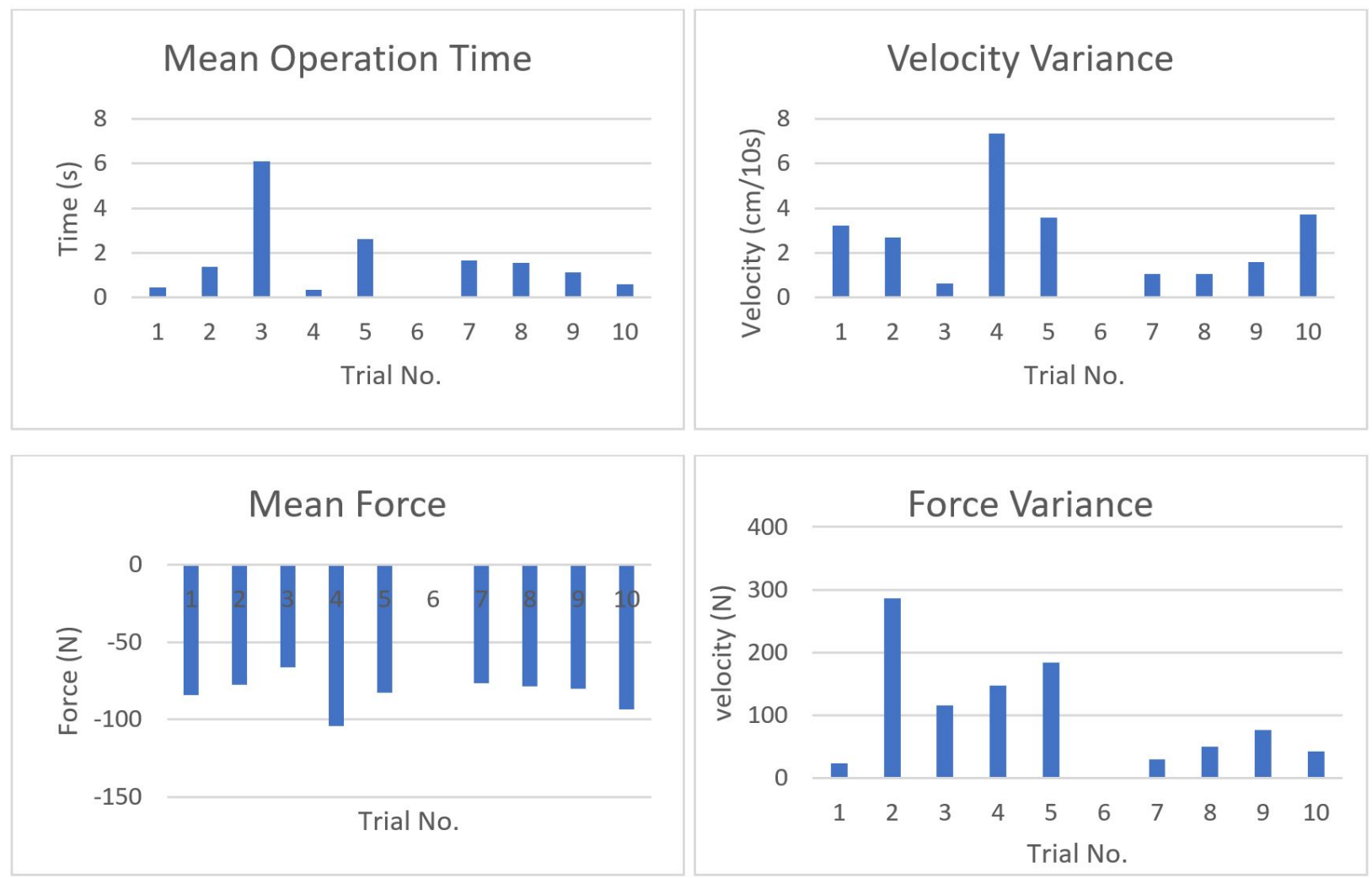

Figure 6.2: Hard probing test results. (Participant 6 did not conduct this test) 


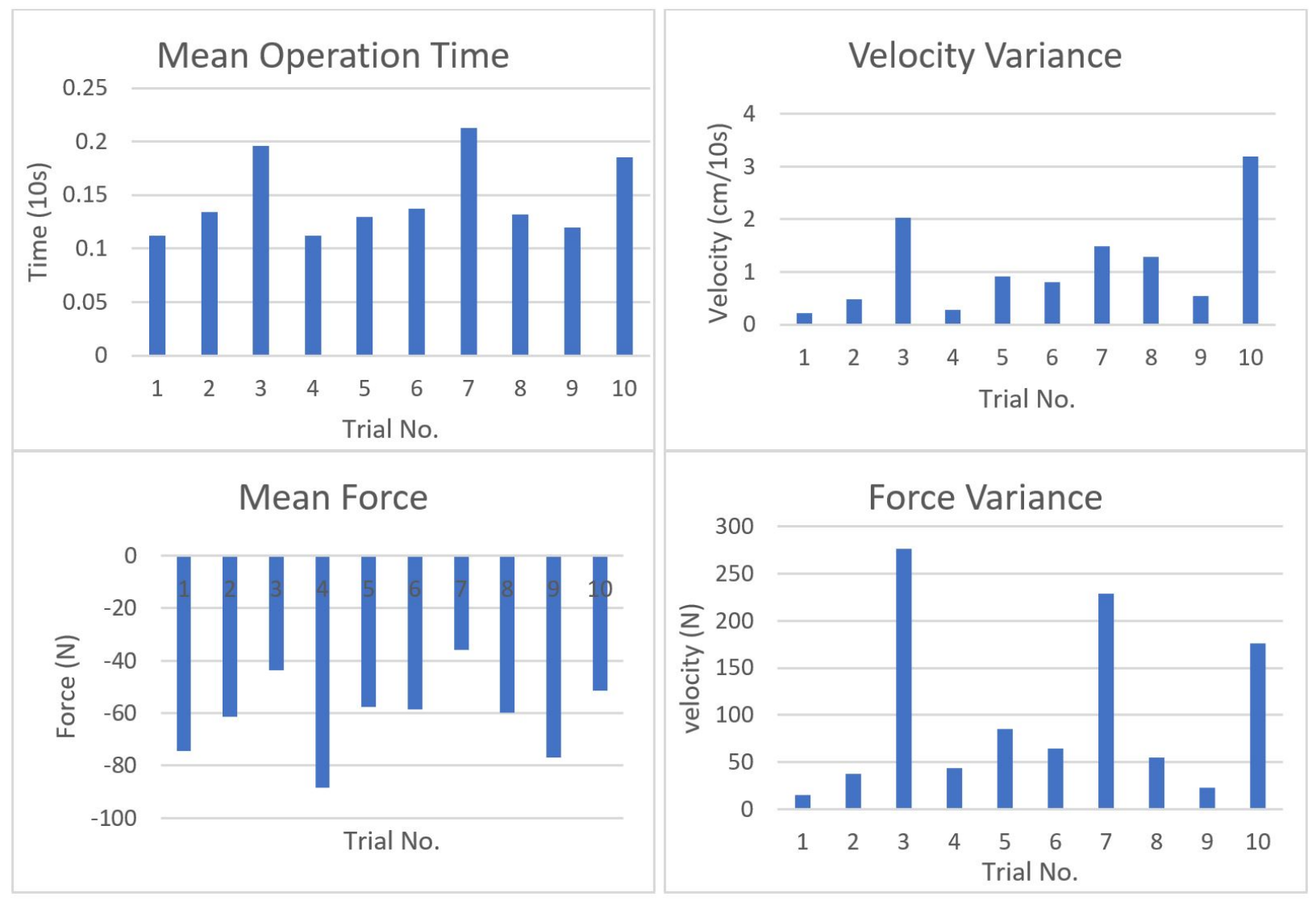

Figure 6.3: Lateral Breach probing test results.

Due to the small sample size, possible outliers may have strong affects on the regression test. A modified Z-score test is conducted before each regression analysis [61]. This is to remove any significant outliers from the data set. The modified Z-score formula is shown as following:

$$
M_{i}=\frac{0.6745\left(x_{i}-\tilde{x}\right)}{\operatorname{median}\left(\left|y_{i}-\tilde{y}\right|\right)}
$$

where $x_{i}$ and $y_{i}$ are the data point at i-th position. $\tilde{x}$ and $\tilde{y}$ are the median of respective set. $M_{i}$ is the Z-score of the tested point. 
A outlier is identified if the absolute value of the modified z-score is bigger than 3.5. In addition to the Z-score test, a M-estimation robust regression was used instead of the linear regression test from the previous research question. Robust regression is a modified linear regression analysis. It is helpful in reducing outlier leverage in normal least square estimation. The M-estimation stands for maximum likelihood estimation. It uses a weighted function to reduce the impact of the outlier. This can lead to a more balanced regression fitting line [62]. The M-estimation robust regression using bisquare estimator can be completed in zathe following steps [63]:

1. A least-square estimation is used to find the initial estimation of $\beta^{(0)}$ [64].

2. For each iteration $\mathrm{t}$, the residual value at $\mathrm{i}$-th point $e_{i}^{(t-1)}$ is calculated using the following equation

$$
e_{i}=y_{i}-\hat{y}_{i}
$$

where $\hat{y}_{i}$ denote the estimation of $\mathrm{Y}$ at i-th point.

3. The tuning constant $\mathrm{k}$ is calculated using the equation below:

$$
k=4.685 * 1.4826 * \operatorname{median}\left(\left|e_{i}-\operatorname{median}\left(e_{i}\right)\right|\right)
$$

4. The weighted value $w_{i}^{(t-1)}=w\left[e_{i}^{(t-1)}\right]$ is calculated using bisquare weighted formula from below:

$$
w= \begin{cases}{\left[1-\left(\frac{e}{k}\right)^{2}\right]^{2}} & \text { for }|e| \leq k \\ 0 & \text { for }|e|>k\end{cases}
$$

5. The new $b^{(t)}$ is then solved using the weighted least square estimate

$$
b^{(t)}=\left[X^{\prime} W^{(t-1)} X\right]^{-1} X^{\prime} W^{(t-1)}
$$


where $\mathrm{X}$ is a matrix of the independent variable $\mathrm{x}$, and $\left.W^{(t-1)}=\operatorname{diag}() w_{i}^{(t-1)}\right)$ is the weight matrix.

6. Steps 2 though 5 is repeated until a convergent estimated coefficient is found.

7. The remaining of the robust regression analysis is conducted using the normal linear regression analysis.

Table 6.5: Robust regression analysis p-value.

\begin{tabular}{|l|l|l|l|}
\hline & Soft Probing & Hard Probing & Lateral Breach \\
\hline Operation Time & 0.5784 & 0.9792 & 0.3269 \\
\hline Velocity Variance & 0.629 & 0.8164 & 0.2707 \\
\hline Force Variance & 0.1049 & $1.34 \mathrm{e}-4$ & 0.3424 \\
\hline Mean Force & 0.9233 & 0.3969 & 0.3838 \\
\hline
\end{tabular}

The Z-score test and robust regression analysis was completed on all three scenarios. The result p-value is shown in Table 6.5. In most of robust regression analysis conducted, no trend or significance was discovered from the data. However, the force variance does provide some interesting results. First, The $p$-value of robust regression analysis for hard probing force variance is $1.34 \mathrm{e}-4$. Because the $p$-value is less than 0.05 , the null hypothesis is rejected. For the soft probing, surgeon 10 is removed from the data set due to high zscore value of 8.72. The $p$-value of the robust regression analysis is 0.1049 . Although this is the above the 0.05 statistically significant level, more data samples could potentially reject the null hypothesis. The results of such a small $p$-value is probably because experienced surgeons are more comfortable with the surgery. They are able to change their applied force according to the simulation haptics feedback. The inexperience surgeons are more focused on completing the task, which means they are more mechanical with less force 
variance. It is also interesting to note that from research question 2, experienced surgeons did not perform better compared to inexperienced surgeons in overall recognition rate. This indicate that improving force variance performance can not help surgeons in better recognition rate. The large variance may prevent surgeon from recognizing the drop in resistance during breach event. It could be interesting to find if lowering expert's force variance can lead to better recognition rate.

\subsubsection{Breach Force Variance}

Experienced surgeons tend to have encountered many different surgical scenarios. They may have a more steady approach to any situation. In this analysis, the standard deviation (STD) of the test data during lateral breach was examined. This is used to find if experience can affect performance under emergency situations. One of the interesting finding is the standard deviation of force variance during lateral breach. The standard deviation is calculated from each surgeon's performance during three trials. Large standard deviation of force variance indicates that the surgeon is approaching the same scenario very differently in three trials.

The data for breach force variation standard deviation is shown in Figure 6.4, where the $\mathrm{x}$-axis is arranged with 1 being the most experienced and 10 being least experienced, the first two surgeons have much smaller force variance change than the rest of the surgeons. One reason to explain this is that both surgeons have at least three times more experience than other surgeons. They are most likely to keep their approach during emergent situations. One way ANOVA(ANalysis Of VAriance) is conducted to find if the null hypothesis is rejected. ANOVA suits this case because it is able compare small groups with different sample size. The surgeons were split into two groups, the first group contains the 
two senior surgeons and the second contains the other eight surgeons. A z-score test is used to remove possible outliers from group 2. Due to the large variation in test results, two data points are identified as outliers. Therefore, they are removed from the analysis. The $p$-value for the ANOVA analysis is 0.0782. Although this is close to 0.05 , it is still not statistically significant, therefore, the null hypothesis is not rejected. Because group 1 only contains two data points, the analysis can only provide limited results. More data can potentially show that experience had effect on lateral breach force variance. Keeping the same approach during surgery can help surgeon better prepare for emergent situations such as breach. Although the better performance by expert surgeon did not lead to better breach recognition rate, the breach force variance STD is still an important factor for the surgery.

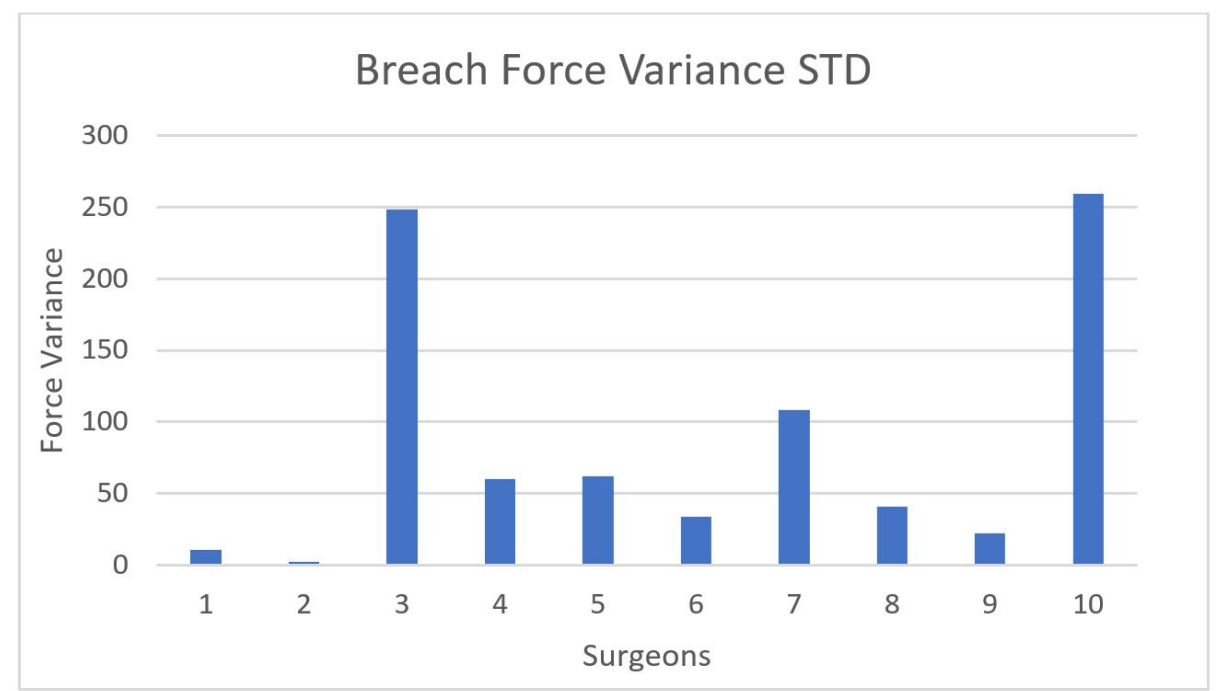

Figure 6.4: Lateral breach force variance standard deviation. 


\subsection{Research Question No. 4}

\section{Can the simulation trials become a learning tool for the simulation tasks?}

$H_{0}$ : Simulation trial cannot be a learning tool for the simulation tasks.

The main goal of the simulator is to help new surgeons prepare for breach event. One effective way is to examine if there are any progress in participant's results over the course of the study. In the analysis for breach event, the data was split into three groups based on each participant's trial sequence. Figure 6.5 illustrates the lateral breach test results. The x-axis is arranged based on surgeon's attempt. Each surgeon's three trials are grouped together and the surgeons are separated by a empty column. The surgeon with trial 1-3 is the most experienced and the surgeon with trial 37-39 is least experienced surgeon. 


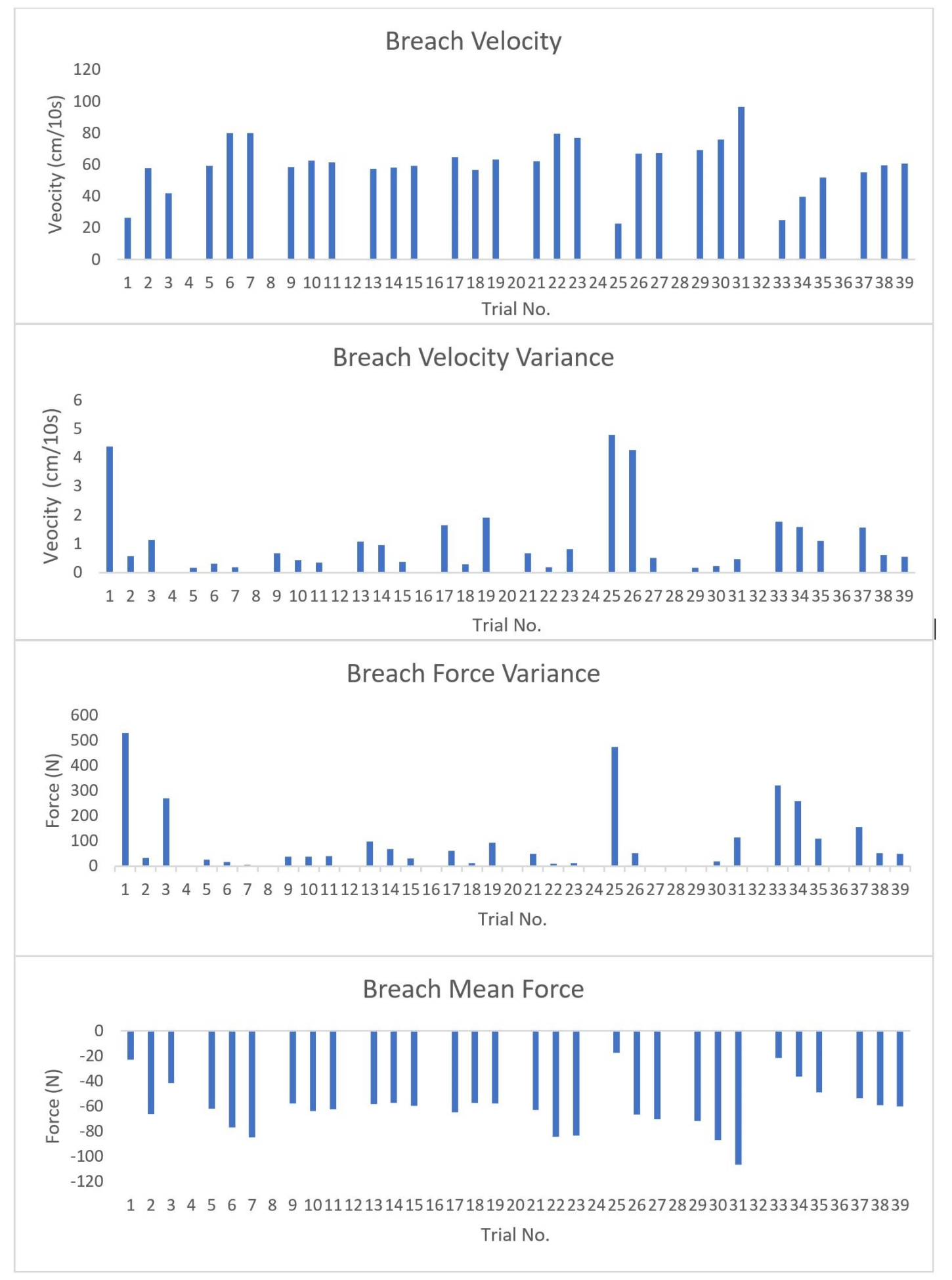

74

Figure 6.5: Lateral breach test results. 
One way ANOVA was used to evaluate between the three trials. ANOVA was chosen because it is able to compare data from three or more groups. The main goal is to find out if surgeons is able learn or adapt during the study and if there is any difference in performance between first, second, and third trial. The resulting p-value is shown in Table 6.6. One of the most importance information for breach is the breach velocity. The $p$-value from ANOVA for breach velocity is 0.0627 , which is close to statistically significant. The $p$-value for breach mean force is at 0.0693 . Because both p-value is above statistically significant of 0.05 , the null hypothesis is not rejected. Although the above two number are not statistically significant, it is interesting to further analyse these data with a larger sample size as they are very close to significant. This potentially can prove there is performance difference from the first trial to the third trial and the simulator is a great learning tool for the tasks.

Table 6.6: One way ANOVA p-value

\begin{tabular}{|l|l|l|l|l|}
\hline & Breach Velocity & Velocity Variance & Force Variance & Mean Force \\
\hline p-value & 0.0627 & 0.2064 & 0.1008 & 0.0693 \\
\hline
\end{tabular}

\subsection{Questionnaire}

At the end of the study, each surgeon is asked to complete a questionnaire with four questions. The questions are intended to be used as a subjective tool to evaluate the simulator performance. It also can be used to find if majority of the participant are satisfied with the two expert surgeons' tuning parameters. 


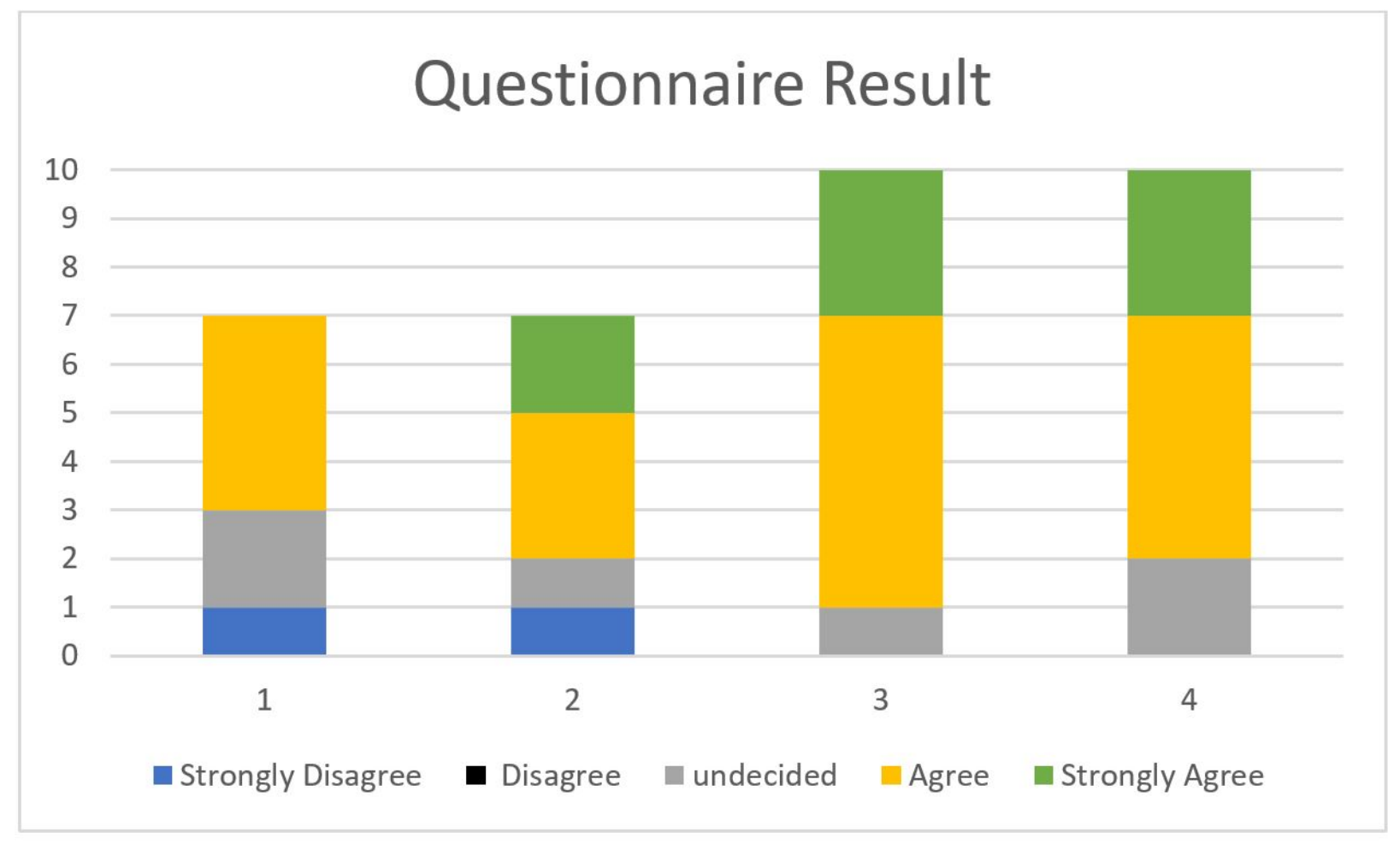

Figure 6.6: Questionnaire result.

The results of the response are shown in Figure 6.6. The x-axis indicates the questions asked and y-axis illustrates the number of responders' pick from each category in the Likert Scale. The questions are listed in the following order 1) The simulator produced realistic haptics sensations during normal probe channelling. 2) The haptics sensation associated with bone breach was simulated realistically. 3) The simulator could potentially be a useful training tool for teaching normal pedicle screw insertion surgery. 4) The simulator could potentially be a useful training tool for bone breach prevention. Due to the large number of residents in this study, many surgeons have not participated in a live patient pedicle 
screw insertion surgery before. Therefore, three participants did not respond to the first two questions. It is important to note that some of the surgeons have experienced breach in similar surgeries, but for simplicity, their response is not included in the response. In total, 7 responses were collected for the first two questions. In evaluation of the results, a benchmark score of $3.5 / 5$ is used. This score indicates that response to the question is positive (3 is neutral). A 90\% confidence interval is used as a measurement tool for the study. The result of the evaluation is shown in Table 6.7.

Table 6.7: Questionnaire result elevation

\begin{tabular}{|c|c|c|c|c|c|}
\hline Question & Benchmark & Mean & $\begin{array}{c}\text { Standard } \\
\text { Deviation }\end{array}$ & $\begin{array}{c}\text { 90\% Confidence } \\
\text { Interval Lower Bound }\end{array}$ & $\begin{array}{c}\text { 90\% Confidence } \\
\text { Interval Upper Bound }\end{array}$ \\
\hline 1 & 3.5 & 3.285714 & 1.112697 & 2.706947 & 3.864482 \\
\hline 2 & 3.5 & 3.714286 & 1.380131 & 2.996413 & 4.432159 \\
\hline 3 & 3.5 & 4.2 & 0.632456 & 3.871029 & 4.528971 \\
\hline 4 & 3.5 & 4.1 & 0.737865 & 3.716201 & 4.483799 \\
\hline
\end{tabular}

The first two questions have a standard deviation of more than 1 and the question score did not reach $90 \%$ confidence interval. This is probably due to the different techniques used by surgeons, while some of the surgeon do agree with the simulator's haptics feedback, many other surgeons may feel different due to their unique rotational or vertical probing technique. For the last two questions, the lower bound of the $90 \%$ probability is above the 3.5 benchmark, this indicates that the response is positive, it shows that the surgeons feel positive for the simulator and it could potentially become a useful training tool for pedicle screw insertion surgery. 


\subsection{Summary}

In this chapter, the four main research questions were discussed. Various test results were presented. The study showed that there is no difference between surgeons with different experience level in breach recognition rate and incorrect recognition rate. Additionally, in the analysis of performance difference between surgeons with difference experience level, the null hypothesis was successfully rejected by analysing force variance. Lastly, the survey questionnaire was analysed and discussed. 


\section{Chapter 7}

\section{Conclusion and Future Works}

In this chapter, the main findings and conclusion are presented. Recommendations for future work of the project are also discussed.

\subsection{Conclusion}

Pedicle screw insertion surgical simulator is a new kind of training simulator. It is able to simulate the hardest and most dangerous part of the pedicle screw insertion surgery, which is the pedicle channel creation process. The main focus of this research is to develop and to test the vertical stage of the simulator. A major emphasis was focused on creating a pedicle breach simulator, which has never been successfully developed. A simulator tuning session was conducted with help of 2 expert surgeons and four scenarios were created to simulate the complex events that can happen in a real surgery. There were 10 other surgeons who were asked to conduct in a clinical study. Measurement was collected for analysis and evaluation. 
The correct breach recognition rate from the 10 surgeons is $85 \%$, and the overall correct recognition rate is $80.8 \%$. By using linear regression analysis on incorrect recognition and incorrect breach recognition with respect to surgeon's experience, it was concluded that there is no statistically significant relationship between the wrong breach recognition rate and surgical experience. In addition, there is no statistically significant relationship between the total wrong recognition rate and surgical experience. However, due to the small sample size, this may be a Type II error.

Further analysis was conducted using the collected measurements. In the hard probing scenarios, it was found that surgical experience has an effect on vertical force variance, where the more experienced surgeons tend to have a larger operating range. However, this may be a Type I error. The soft probing's force variance is also very close to statistically significant. With help of more data, it may be possible to reject the null hypothesis. Next, by splitting surgeons into two groups, it was found that the two expert surgeons have significantly less force variance during breach. One way ANOVA was conducted, and result was very close to statistically significant. All these analyses require more data to avoid Type II error.

The difference between the trials of the same scenario were also analysed. This was used to evaluate whether the simulator is an effective training tool and if the surgeons did adapt over the study time. One of the most important indicator for this analysis is the breach data. By using ANOVA, it was found that the p-value for breach velocity is 0.0627. The p-value for breach mean force is at 0.0693 . Although both are not considered statistically significant, they are close and could be interesting to further analyse the data with a larger sample size. If the result is approved to be significant with more data, it is important note it may still be a Type I error.

On the survey questionnaire side, most of the surgeons have agreed that the simulator 
could be a useful training tool for the future. From the limited feedback, the simulator requires more tuning from different surgeons to account for different techniques used by surgeons.

\section{$7.2 \quad$ Future work}

This project should continue collecting data from more surgeons. Currently, there are only 10 participants conducted the clinical study. Many analyses such as the breach velocity regression analysis are close to statistically significant. A larger sample size could potentially help reject the null hypothesis. Furthermore, the larger sample size could lead to new finding that were previously ignored due to Type I error. Lastly, it is interesting to re-conduct the study with the participants after a certain period of time. Since many participating surgeons currently have minimum experience in the pedicle screw insertion surgery, it may be interesting to find if there is any progression or regression in their surgical skill after a period of study in pedicle screw insertion.

The current haptics sensation tuning and scenario design was only completed by two expert surgeons. From the clinical study, it was clear that many surgeons operate differently, and even expert surgeons can have significant different operating techniques. The simulator could be greatly improved if more expert surgeons were involved in the tuning process. This can lead to creation of a universal scenario.

Another way to help improve the simulator is to change the mechanical set-up. Currently, the rotary stage is on top of the vertical stage. Because the rotary stage is much heavier than the vertical stage, it puts a lot of unnecessary stress on the vertical actuator. This made the linear actuator less reliable. As a result, the linear actuator acceleration was limited. By redesigning and exchanging the two stage's position, the linear actuator 
can become much faster, this possibly can lead to possibility of more simulation scenarios. Due to the high power of the rotary motor, the rotary stage's performance should not have any ill effect.

\subsubsection{Virtual Project System}

In order to improve the realism of the actuator, a 3D projected virtual simulation was developed on the 3D printed torso. This can help surgeons in visualizing the surgery. A 3D image projection system was developed using TouchDesigner [65]. The system uses two projectors to project 3D surgical images on to the torso. This creates a realistic surgical environment for the surgeons. An illustration of such an image is shown in Figure 7.1.

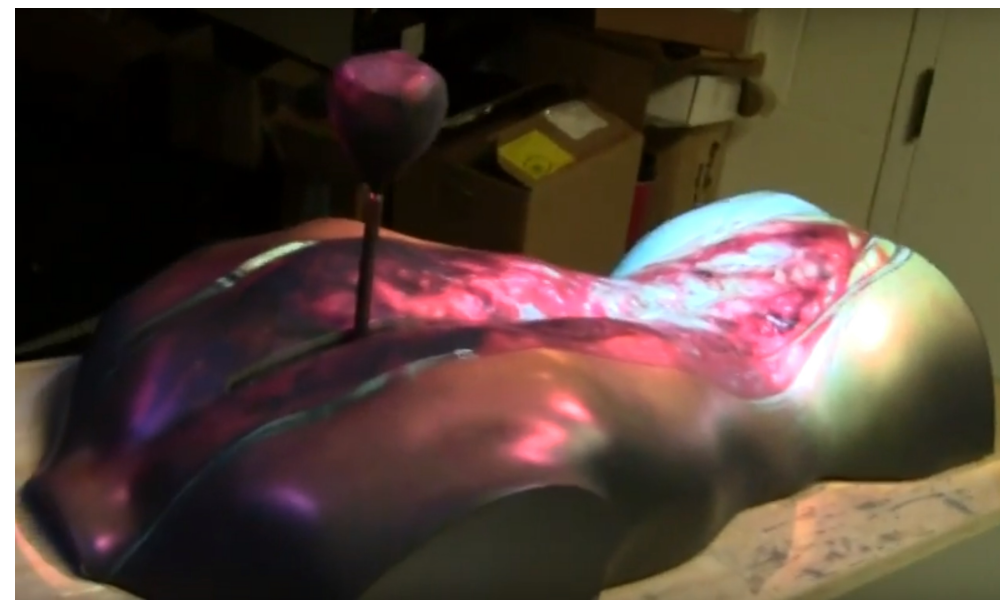

Figure 7.1: 3D image project on the torso [12]

Although this system provides many advantages to the simulation, there are a few drawbacks. The biggest drawback is that the projector is unable to avoid projecting on outside objects such as the surgeon's hand. This can be problematic for some surgeons as it gives a false illusion of the situation. The other drawback is lack of lighting in the projected 
environment. This is especially a problem as surgeons are used to will-lit operation rooms. Due to these drawbacks, the projection system was not used for the study.

Developing a tracking and exclusion algorithm can significantly improves the realism of the simulator. This allows the surgeon to operate on the simulator without misleading views. It may also lead to development in other surgical simulations and possibly even in robot assisted surgeries. 


\section{References}

[1] Questions and answers about scoliosis in children and adolescents. [Online]. Available: https://www.niams.nih.gov/Health_Info/Scoliosis/default.asp

[2] The vertebral column, spinal cord, and neck. [Online]. Available: https: //basicmedicalkey.com/the-vertebral-column-spinal-cord-and-neck-2/

[3] Scoliosis. [Online]. Available: http://muscleandjoint.ca/scoliosis/

[4] Pedicle screw fixation. [Online]. Available: https://www.linkedin.com/pulse/ pedicle-screw-fixation-howard-cotler-md-facs-fabos/

[5] Scoliosis. [Online]. Available: http://www.bcchildrens.ca/our-services/clinics/ orthopaedics/spine-conditions/scoliosis

[6] Adolescent idiopathic scoliosis. [Online]. Available: https://www2. aofoundation.org/wps/portal/!ut/p/a1/04_Sj9CPykssy0xPLMnMz0vMAfGjzOKN_ A0M3D2DDbz9_UMMDRyDXQ3dw9wMDAwCTYEKIvEocDQnTr8BDuBoQEh_ QW5oKABaevup/dl5/d5/L2dJQSEvUUt3QS80SmlFL1o2XzJPMDBHSVMw S09PVDEwQVNFMUdWRjAwMFE1/?approach=\&bone=Spine\&classification= \&implantstype $=\&$ method $=\&$ redfix_url $=\&$ segment $=$ DeformityScoliosis\&showPage $=$ redfix\&treatment $=$ 
[7] Percutaneous pedicle screws. [Online]. Available: http://www.tjkleemanmd.com/ pediclescrews.html

[8] J. Brewin, T. Nedas, B. Challacombe, O. Elhage, J. Keisu, and P. Dasgupta, "Face, content and construct validation of the first virtual reality laparoscopic nephrectomy simulator," BJU international, vol. 106, no. 6, pp. 850-854, 2010.

[9] S. Moafimadani, "Haptic training simulator for pedicle screw insertion in scoliosis surgery," Master's Thesis, University of Waterloo.

[10] R. Leung, "Design of a haptic simulator for pedicle screw insertion in pediatric scoliosis surgery," Master's thesis, University of Toronto.

[11] Original line electric actuator. [Online]. Available: http://www.bimba.com/ ImageVaultFiles/id_223/cf_12/OLE.JPG

[12] Pedicle screw insertion. [Online]. Available: https://www.youtube.com/watch?v= _CIj0KMXt7c\&feature=youtu.be

[13] J. D. Cassidy, L. J. Carroll, and P. Côté, "The saskatchewan health and back pain survey: the prevalence of low back pain and related disability in saskatchewan adults," Spine, vol. 23, no. 17, pp. 1860-1866, 1998.

[14] L. Manchikanti, V. Singh, S. Datta, S. P. Cohen, and J. A. Hirsch, "Comprehensive review of epidemiology, scope, and impact of spinal pain." Pain physician, vol. 12, no. 4, pp. E35-70, 2008.

[15] Scoliosis. [Online]. Available: http://www.hopkinsmedicine.org/healthlibrary/ conditions/orthopaedic_disorders/scoliosis_85,P07815/ 
[16] Scoliosis: What you need to know. [Online]. Available: https://www.spine-health. com/conditions/scoliosis/scoliosis-what-you-need-know

[17] A. Sud, A. I. Tsirikos et al., "Current concepts and controversies on adolescent idiopathic scoliosis: Part i," Indian journal of orthopaedics, vol. 47, no. 2, p. 117, 2013.

[18] J. M. Hicks, A. Singla, F. H. Shen, and V. Arlet, "Complications of pedicle screw fixation in scoliosis surgery: a systematic review," Spine, vol. 35, no. 11, pp. E465E470, 2010.

[19] T. Mattei, M. Meneses, J. Milano, and R. Ramina, "'Free-hand" technique for thoracolumbar pedicle screw instrumentation: Critical appraisal of current "State-of-Art"," Neurology India, vol. 57, no. 6, pp. 715-721, 2009. [Online]. Available: http://www.neurologyindia.com/article.asp?issn=0028-3886;year=2009; volume $=57 ;$ issue $=6 ;$ spage $=715 ;$ epage $=721 ;$ aulast $=$ Mattei $; \mathrm{t}=6$

[20] J. E. Lonstein, F. Denis, J. H. Perra, M. R. Pinto, M. D. Smith, and R. B. Winter, "Complications associated with pedicle screws," J Bone Joint Surg Am, vol. 81, no. 11, pp. 1519-28, 1999.

[21] J. Silbermann, F. Riese, Y. Allam, T. Reichert, H. Koeppert, and M. Gutberlet, "Computer tomography assessment of pedicle screw placement in lumbar and sacral spine: comparison between free-hand and o-arm based navigation techniques," European Spine Journal, vol. 20, no. 6, pp. 875-881, 2011.

[22] Y. J. Kim, L. G. Lenke et al., "Thoracic pedicle screw placement: free-hand technique," Neurology India, vol. 53, no. 4, p. 512, 2005.

[23] B. J. Shin, A. R. James, I. U. Njoku, and R. Härtl, "Pedicle screw navigation: a systematic review and meta-analysis of perforation risk for computer-navigated versus 
freehand insertion: A review," Journal of Neurosurgery: Spine, vol. 17, no. 2, pp. 113$122,2012$.

[24] I. D. Gelalis, N. K. Paschos, E. E. Pakos, A. N. Politis, C. M. Arnaoutoglou, A. C. Karageorgos, A. Ploumis, and T. A. Xenakis, "Accuracy of pedicle screw placement: a systematic review of prospective in vivo studies comparing free hand, fluoroscopy guidance and navigation techniques," European Spine Journal, vol. 21, no. 2, pp. $247-255,2012$.

[25] H. Smith, M. Welsch, H. Ugurlu, R. Sasso, and A. Vaccaro, "Comparison of radiation exposure in lumbar pedicle screw placement with fluoroscopy vs computer-assisted image guidance with intraoperative three-dimensional imaging," The Journal of Spinal Cord Medicine, vol. 31, no. 5, pp. 532-537, 2008. [Online]. Available: http://dx.doi.org/10.1080/10790268.2008.11753648

[26] O. Safety, H. Administration et al., "Ionizing radiation-1910.1096," US Dept. of Labor, Washington, DC, 1996.

[27] A. A. Aoude, M. Fortin, R. Figueiredo, P. Jarzem, J. Ouellet, and M. H. Weber, "Methods to determine pedicle screw placement accuracy in spine surgery: a systematic review," European Spine Journal, vol. 24, no. 5, pp. 990-1004, 2015.

[28] W. Sukovich, S. Brink-Danan, and M. Hardenbrook, "Miniature robotic guidance for pedicle screw placement in posterior spinal fusion: early clinical experience with the spineassist@," The International Journal of Medical Robotics and Computer Assisted Surgery, vol. 2, no. 2, pp. 114-122, 2006.

[29] F. Ringel, C. Stüer, A. Reinke, A. Preuss, M. Behr, F. Auer, M. Stoffel, and B. Meyer, "Accuracy of robot-assisted placement of lumbar and sacral pedicle screws: a prospec- 
tive randomized comparison to conventional freehand screw implantation," Spine, vol. 37, no. 8, pp. E496-E501, 2012.

[30] A. F. Samdani, A. Ranade, D. M. Sciubba, P. J. Cahill, M. D. Antonacci, D. H. Clements, and R. R. Betz, "Accuracy of free-hand placement of thoracic pedicle screws in adolescent idiopathic scoliosis: how much of a difference does surgeon experience make?" European Spine Journal, vol. 19, no. 1, pp. 91-95, 2010.

[31] R. A. Lehman Jr, L. G. Lenke, K. A. Keeler, Y. J. Kim, and G. Cheh, "Computed tomography evaluation of pedicle screws placed in the pediatric deformed spine over an 8-year period," Spine, vol. 32, no. 24, pp. 2679-2684, 2007.

[32] Y. J. Kim, L. G. Lenke, K. H. Bridwell, Y. S. Cho, and K. D. Riew, "Free hand pedicle screw placement in the thoracic spine: is it safe?" Spine, vol. 29, no. 3, pp. 333-342, 2004.

[33] R. Zeller, "Scoliosis surgery experiment and test data." [Online]. Available: http://www.sickkids.ca/AboutSickKids/Directory/People/Z/Reinhard-Zeller.html

[34] T. R. Coles, D. Meglan, and N. W. John, "The role of haptics in medical training simulators: A survey of the state of the art," IEEE Transactions on haptics, vol. 4, no. 1, pp. 51-66, 2011.

[35] J. Vozenilek, J. S. Huff, M. Reznek, and J. A. Gordon, "See one, do one, teach one: advanced technology in medical education," Academic Emergency Medicine, vol. 11, no. 11, pp. 1149-1154, 2004.

[36] A. Gonzalvo, G. Fitt, S. Liew, D. de la Harpe, P. Turner, L. Ton, M. A. Rogers, and P. H. Wilde, "The learning curve of pedicle screw placement: how many screws are enough?" Spine, vol. 34, no. 21, pp. E761-E765, 2009. 
[37] M. Akamatsu, I. S. MacKenzie, and T. Hasbroucq, "A comparison of tactile, auditory, and visual feedback in a pointing task using a mouse-type device," Ergonomics, vol. 38, no. 4, pp. 816-827, 1995.

[38] R. L. Klatzky, S. J. Lederman, and C. Reed, "There's more to touch than meets the eye: The salience of object attributes for haptics with and without vision." Journal of experimental psychology: general, vol. 116, no. 4, p. 356, 1987.

[39] M. A. Srinivasan and C. Basdogan, "Haptics in virtual environments: Taxonomy, research status, and challenges," Computers \& Graphics, vol. 21, no. 4, pp. 393-404, 1997.

[40] N. E. Seymour, A. G. Gallagher, S. A. Roman, M. K. Obrien, V. K. Bansal, D. K. Andersen, and R. M. Satava, "Virtual reality training improves operating room performance: results of a randomized, double-blinded study," Annals of surgery, vol. 236, no. 4, pp. 458-464, 2002.

[41] F. Carter, M. Schijven, R. Aggarwal, T. Grantcharov, N. Francis, G. Hanna, and J. Jakimowicz, "Consensus guidelines for validation of virtual reality surgical simulators," Surgical Endoscopy and Other Interventional Techniques, vol. 19, no. 12, pp. $1523-1532,2005$.

[42] K. Tanoue, S. Ieiri, K. Konishi, T. Yasunaga, K. Okazaki, S. Yamaguchi, D. Yoshida, Y. Kakeji, and M. Hashizume, "Effectiveness of endoscopic surgery training for medical students using a virtual reality simulator versus a box trainer: a randomized controlled trial," Surgical endoscopy, vol. 22, no. 4, pp. 985-990, 2008. 
[43] R. Aggarwal, J. Ward, I. Balasundaram, P. Sains, T. Athanasiou, and A. Darzi, "Proving the effectiveness of virtual reality simulation for training in laparoscopic surgery," Annals of surgery, vol. 246, no. 5, pp. 771-779, 2007.

[44] D. L. Dawson, J. Meyer, E. S. Lee, and W. C. Pevec, "Training with simulation improves residents endovascular procedure skills," Journal of vascular surgery, vol. 45, no. 1, pp. 149-154, 2007.

[45] G. J. Wiet, D. Stredney, D. Sessanna, J. A. Bryan, D. B. Welling, and P. Schmalbrock, "Virtual temporal bone dissection: an interactive surgical simulator," OtolaryngologyHead and Neck Surgery, vol. 127, no. 1, pp. 79-83, 2002.

[46] A. George and R. De, "Review of temporal bone dissection teaching: how it was, is and will be," The Journal of Laryngology \& Otology, vol. 124, no. 02, pp. 119-125, 2010.

[47] M. Vankipuram, K. Kahol, A. McLaren, and S. Panchanathan, "A virtual reality simulator for orthopedic basic skills: A design and validation study," Journal of biomedical informatics, vol. 43, no. 5, pp. 661-668, 2010.

[48] A. Manbachi, R. S. Cobbold, and H. J. Ginsberg, "Guided pedicle screw insertion: techniques and training," The Spine Journal, vol. 14, no. 1, pp. 165-179, 2014.

[49] S. Klein, C. M. Whyne, R. Rush, and H. J. Ginsberg, "Ct-based patient-specific simulation software for pedicle screw insertion," Clinical Spine Surgery, vol. 22, no. 7, pp. 502-506, 2009.

[50] C. J. Luciano, P. P. Banerjee, J. M. Sorenson, K. T. Foley, S. A. Ansari, S. Rizzi, A. V. Germanwala, L. Kranzler, P. Chittiboina, and B. Z. Roitberg, "Percutaneous 
spinal fixation simulation with virtual reality and haptics," Neurosurgery, vol. 72, pp. A89-A96, 2013.

[51] Sawbones. [Online]. Available: https://www.sawbones.com/

[52] J. Gasco, A. Patel, J. Ortega-Barnett, D. Branch, S. Desai, Y. F. Kuo, C. Luciano, S. Rizzi, P. Kania, M. Matuyauskas et al., "Virtual reality spine surgery simulation: an empirical study of its usefulness," Neurological research, vol. 36, no. 11, pp. 968-973, 2014.

[53] Q. Xing, J. X. Chen, J. Li, A. Moshirfar, M. M. Theiss, and Q. Wei, "A real time haptic simulator of spine surgeries," in Proceedings of the 21st ACM Symposium on Virtual Reality Software and Technology. ACM, 2015, pp. 121-124.

[54] J.-J. Fang, C.-Y. Yang, and R.-M. Lin, "Development of a computer-aided training system for pedicle screw implantation," Biomedical Engineering: Applications, Basis and Communications, vol. 19, no. 05, pp. 323-329, 2007.

[55] Bimba actuator. [Online]. Available: http://www.bimba. com/Products-and-Cad/Electric-Actuators/Inch/Rod-Style/Standard-Duty/ Original-Line-Electric-Actuator/

[56] C. Ott, R. Mukherjee, and Y. Nakamura, "Unified impedance and admittance control," in 2010 IEEE International Conference on Robotics and Automation, May 2010, pp. $554-561$.

[57] Reinhard. [Online]. Available: http://www.sickkids.ca/AboutSickKids/Directory/ People/Z/Reinhard-Zeller.html 
[58] Dr. ryan o'shea. [Online]. Available: https://health.usnews.com/doctors/ ryan-oshea-1123399

[59] G. A. Seber and A. J. Lee, Linear regression analysis. John Wiley \& Sons, 2012, vol. 936.

[60] S. Goodman, "A dirty dozen: Twelve p-value misconceptions," Semin Hematol, vol. 45 , pp. $135-140$.

[61] M. Natrella, "Nist/sematech e-handbook of statistical methods," 2010.

[62] A. Ruckstuhl, "Robust fitting of parametric models based on m-estimation," Lecture notes.[Available at https://stat. ethz. ch/wbl/wbl4/WBL4_robstat14E. pdf.], 2014.

[63] J. Fox, "Robust regression," An R and S-Plus companion to applied regression, 2002.

[64] Y. Susanti, H. Pratiwi et al., "M estimation, s estimation, and $\mathrm{mm}$ estimation in robust regression," International Journal of Pure and Applied Mathematics, vol. 91, no. 3, pp. 349-360, 2014.

[65] Derivative. [Online]. Available: https://www.derivative.ca/ 
APPENDICES 


\section{Appendix A}

\section{Hardware Components}

\section{Computer}

A Desktop computer with Intel Sandy Bridge Core i7 processor (Clocked at $3.4 \mathrm{GHz}$ ) and 8GB RAM is used for the simulator. The operating system is Windows 7.

\section{Data Acquisition}

A Sensoray Model 626 PCI data acquisition card coupled with a MultiQ-PCI terminal board is used for the rotary stage. The DAQ is responsible for acquiring the input and send output to the amplifier and the rotary servo motor.

\section{Amplifier}

An AMC 50AI Brush Type PWM Servo Amplifier is used to drive the rotary motor. The amplifier is set to current mode with a gain of 1 . The amplifier is powered by a $57 \mathrm{~V}$ DC power supply.

\section{Rotary motor}

A Kollmorgen JR16M4CH ServoDisc Motor is used for the rotary stage. 


\section{Encoder}

A SICK DFS60 (model DFS60A-S4AC65536) encoder is used to measure angular position of the probe.

\section{Force/Torque Sensor}

An ATI IP68 Nano25 is used to measure the force and torque acted on the probe. An ATI DAQ F/T is used to acquire the data.

\section{Linear Actuator}

A Bimba OLE-1507-25Q-E2 is used for the vertical stage. The actuator contains an encoder which can be used to measure the actuator's rotation movements. The actuator is driven by a Bimba amplifier with $48 \mathrm{~V}$ DC power supply. The amplifier is set to velocity mode.

\section{Position Sensor}

A Celesco SP2 string potentiometer is used to measure the vertical movement of the simulator.

\section{$3 \mathrm{D}$ torso}

A 3D printed torso is used to provide visual aid for the simulator. The torso contains only the upper body. 